\title{
Leucine and arginine regulate trophoblast motility through mTOR-dependent and independent pathways in the preimplantation mouse embryo
}

\author{
Isabel M. González ${ }^{1}$, Patrick M. Martin ${ }^{2}$, Carol Burdsal ${ }^{3}$, Jennifer L. Sloan ${ }^{4,5}$, Sela Mager ${ }^{4}$, \\ Thurl Harris $^{6}$, and Ann E. Sutherland ${ }^{1,7}$ \\ ${ }^{1}$ Department of Cell Biology, University of Virginia Health System, PO Box 800732, \\ Charlottesville, VA 22901; email for IMG is img3v@virginia.edu, email for AES is \\ as9n@virginia.edu \\ ${ }^{2}$ Biology Department, 104 Barnes Hall, North Carolina A\&T State University, 1601 E. Market \\ Street, Greensboro, NC 27411; pmmartin@ncat.edu \\ ${ }^{3}$ Department of Cell and Molecular Biology, School of Science and Engineering, Tulane \\ University, New Orleans, LA; cburdsal@nsf.gov \\ ${ }^{4}$ Department of Cell and Molecular Physiology, University of North Carolina at Chapel Hill, 630 \\ Medical Biomolecular Research Building, Campus Box 7545, Chapel Hill, NC 27599-7545, USA \\ ${ }^{6}$ Department of Pharmacology, University of Virginia Health System, Charlottesville, Virginia, \\ USA; teh3c@virginia.edu
}

\section{Introduction}

Successful uterine implantation is a critical element of mammalian reproduction, without which the embryo cannot continue its development. The process of implantation is a tightly and highly coordinated event that is systematically and systemically driven by changes in the hypothalamic-pituitary-gonadal axis (Belchetz et al., 1978; Bliss et al., 2010; Levine et al., 1982; Levine and Ramirez, 1982; Midgley and Jaffe, 1971; Santen and Bardin, 1973). Cyclic changes in the ovarian derived hormones progesterone (P4) and estrogen (E2) not only direct follicular maturation and ovulation, but significantly, also cause changes to uterine physiology that alter its normally non-receptive state to a environment receptive to the conceptus (Chen et al., 2000; Das et al., 1994b; Lubahn et al., 1993; Lydon et al., 1995; Psychoyos, 1973a, b; Song et al., 2000; Tranguch et al., 2005; Wang et al., 1994; Yoshinaga and Adams, 1966; Yoshinaga et al., 1969). This receptive state lasts for a very short time during the estrus cycle, and is thus defined as a "window of receptivity".

Concomitantly, but in an initially separate process, the mouse embryo follows an intrinsic differentiation program. After fertilization the zygote increases in cell number, initiates

\footnotetext{
(c) 2011 Elsevier Inc. All rights reserved.

${ }^{7}$ Author for correspondence: Ann E. Sutherland, Department of Cell Biology, University of Virginia, 1300 Jefferson Park Ave., PO Box 800732, Charlottesville, VA, Telephone: 434-243-6711, FAX: 434-982-3912, as9n@ virginia.edu.

${ }^{5}$ Current address: Genetic and Molecular Biology Branch, National Human Genome Research Institute, National Institutes of Health, Bethesda, Maryland, USA; jsloan@mail.nih.gov

Publisher's Disclaimer: This is a PDF file of an unedited manuscript that has been accepted for publication. As a service to our customers we are providing this early version of the manuscript. The manuscript will undergo copyediting, typesetting, and review of the resulting proof before it is published in its final citable form. Please note that during the production process errors may be discovered which could affect the content, and all legal disclaimers that apply to the journal pertain.
} 
zygotic gene transcription, and undergoes two integral morphogenetic events: blastomere compaction and blastocoel formation (Biggers et al., 1977; Ziomek and Johnson, 1980). As a result, the formerly spherical embryo becomes a blastocyst comprised of the inner cell mass and an encompassing layer of trophectoderm. The inner cell mass will give rise to the embryo proper, while the trophectoderm will initiate implantation and give rise to the extraembryonic structures (Hillman et al., 1972; Tarkowski and Wroblewska, 1967). Once formed, the blastocyst will undergo a process of activation consisting of physiological changes that enable it to attach to the uterine epithelium and invade into the underlying stroma (Carson et al., 1993; Das et al., 1994b; Hamatani et al., 2004; Paria et al., 1993b; Raab et al., 1996; Schultz and Armant, 1995; Schultz et al., 1997; Wang et al., 2000; Xie et al., 2008).

For implantation to occur properly, the change in uterine receptivity and the activation of the blastocyst must be very precisely coordinated. To accomplish this, the embryo and uterus exist is in a state of continuous crosstalk consisting of signals that regulate each other's progress toward implantation. Disruption of this embryo-uterine dialogue disrupts the process of implantation, diminishes fertility, and causes adverse ripple effects on placentation and offspring viability (Song et al., 2002; Wang et al., 2004; Wang et al., 2007; Wilcox et al., 1999; Ye et al., 2005).

Uterine receptivity in the mouse is initiated by a surge of nidatory E2 on the morning of implantation which induces changes in gene expression and, in particular, expression of the cytokine Leukemia Inhibitory Factor (LIF) in the glandular epithelium (Bhatt et al., 1991; Shen and Leder, 1992; Stewart et al., 1992). LIF is the key regulator of receptivity downstream of E2 signals, and is sufficient to trigger implantation in the absence of an E2 surge (Chen et al., 2000). It is a potent intercellular signaling molecule, with pleiotropic effects on both uterine epithelium and stroma that are necessary for stromal cell decidualization (Chen et al., 2000; Fouladi-Nashta et al., 2005), as well as for regulating blastocyst activation and embryonic gene and protein expression (Hamatani et al., 2004). The molecular pathways regulating uterine receptivity downstream of E2 and LIF have begun to be elucidated, with the progesterone receptor (PR) and Indian Hedgehog (Ihh) identified as key mediators of the receptivity response both in the epithelium (Franco et al., 2010; Lee et al., 2006; Wakitani et al., 2008) and in the stroma (Franco et al., 2011; Large and Demayo, 2011; Lydon et al., 1995). The importance of LIF, IHH, and PR to uterine receptivity and blastocyst activation is demonstrated by the findings that mice lacking any of these proteins are infertile due to failure of blastocyst attachment and decidualization.

While progress has been made in understanding the regulation of uterine receptivity, the process of blastocyst activation itself remain poorly understood. Previous work indicates that amino acids (AA) play an important role in blastocyst activation. Mouse embryos cultivated in vitro require a specific and defined culture medium containing amino acids (Gwatkin, 1966; Naeslund, 1979; Spindle, 1980; Spindle and Pedersen, 1973). This embryonic requirement for AA is not simply nutritive, as AA exposure induces trophectoderm motility through activation of mTOR-dependent signal transduction cascades only after the embryo has reached the early blastocyst stage (Martin and Sutherland, 2001; Martin et al., 2003). A short pulse (4-8 hr) of AA exposure is sufficient to induce motility in the trophectoderm of the early blastocyst, leading to trophoblast outgrowth in an in vitro model of in vivo implantation behavior (Martin and Sutherland, 2001; Martin et al., 2003). The requirement for AA at this stage acts as a developmental checkpoint; when cultured in vitro without AA supplementation, blastocysts remain in a quiescent state, from which trophoblast motility can be induced at any time by adding AA to the culture medium. Once induced, the effect of AA is not reversible, as removing AA will not then switch embryos back to a quiescent state. 
Through an as yet incompletely defined mechanism, AA have long been known to regulate the activity of mTOR (mammalian target of rapamycin); a highly conserved serine/threonine protein kinase which phosphorylates specific downstream effectors and in turn promotes overall cellular growth via the translation of specific populations of mRNAs. mTOR is known to exist in two biochemically and functionally distinct protein complexes: mTORC1 and mTORC2 (Hara et al., 2002; Kim et al., 2002; Sarbassov et al., 2004), which have distinct downstream targets and cellular effects. mTORC1 signaling is required for mouse embryo implantation (Gangloff et al., 2004; Guertin et al., 2006), and has been implicated in AA regulation of trophoblast motility (Martin and Sutherland, 2001). However, the pathways linking amino acids to mTORC1 and then to motility have not been identified. We show here that leucine and arginine, and in particular, uptake of leucine through the SLC6A14 amino acid transporter, are each required individually and together are sufficient to induce blastocyst activation. Importantly, leucine and arginine activate mTORC1 and are rapamycin-sensitive, both individually and in combination, yet activate TE motility independently of the well-characterized targets of mTORC1, p70S6K and 4EBP. Blastocyst activation in vivo is accompanied by changes in mTOR localization, and these changes occur on day 4.5 of gestation, after the onset of embryo attachment, suggesting that there are two separate signals from the uterus regulating the phases of implantation.

\section{Materials and Methods}

\section{Materials}

ICR male and female mice were obtained from Taconic. Embryo culture medium (Martin and Sutherland, 2001; Spindle, 1980; Stephens et al., 1995) was produced in house using embryo tested essential amino acids, salts, bovine serum albumin (BSA), and mineral oil purchased from Sigma Aldrich Chemicals (St. Louis, MO). Antibodies used for detection of total and phosphorylated proteins via western blots against phospho-p70S6K Thr389 (\#9205), p70S6K Total (\#9202), phospho-4EBP 37/46 (\#9459), and 4EBP total (\#9452) were purchased from Cell Signaling Technology (Boston, MA). Phospho-4EBP Ser 65 was produced by Dr. Thurl Harris (University of Virginia). Antibodies used for protein expression and localization of mTOR (\#2972), Raptor (\#24C12), Rictor (\#53A2), and LST8 (\#86B8) by western blot and immunofluorescence were also purchased from Cell Signaling Technology. The antibody used to detect protein levels of SLC6A14 (\#ab99102) via immunoblot was purchased from Abcam (Cambridge, MA) and antibody to tubulin was from Sigma. Bovine fibronectin, cycloheximide, NO711, BCH, and DMSO were purchased from Sigma, and rapamycin was from Calbiochem (San Diego, CA). The antibodies used for mTOR immunofluorescence were purchased from Cell Signaling Technology (\#2983) and Santa Cruz Biotechnology (\#SC-136269) The superovulatory hormone PMSG was purchased from CalBiochem and hCG was from Sigma. $\left[{ }^{3} \mathrm{H}\right]$ Arginine and $\left[{ }^{3} \mathrm{H}\right]$ leucine were purchased from Perkin Elmer (Waltham, MA).

\section{Embryo Culture}

Embryos were flushed at the 2-cell stage from superovulated and mated female ICR mice and cultured from 48 to $120 \mathrm{~h}$ phCG as previously described (Martin and Sutherland, 2001; Sutherland et al., 1993). 120h phCG embryos were then transferred to $10 \mu 1$ drops of serum free medium supplemented with $4 \mathrm{mg} / \mathrm{ml} \mathrm{BSA}$ and amino acids and cultured for an additional 8-48 hours depending on experimental design. After amino acid exposure embryos were subjected to a trophoblast cell spreading assay. Medium containing a complete mixture of amino acids will be designated $+\mathrm{AA}$, and medium with no amino acids -AA. - Arg, - Leu, or -Arg/ - Leu refers to medium excluding only arginine and/or leucine individually while retaining all other amino acids. + Arg, + Leu or + Arg/+Leu refers to medium containing only $0.2 \mathrm{mM}$ arginine and or leucine and no other amino acids. 
Rapamycin (RAPA 25nM) was used to inhibit mTOR, cycloheximide (CHX 50mg/ml) was used to inhibit protein translation, and $\alpha$-amanitin $(10 \mathrm{mg} / \mathrm{ml})$ was used to inhibit transcription. BCH and NO711 were used at a concentration of $100 \mathrm{mM}$ to inhibit SLC6A14 activity. All inhibitors were prepared from stock solutions in appropriate chemical vehicles (DMSO or water) and then diluted in medium immediately prior to use.

\section{Trophoblast Cell Spreading Assay}

Substrates of bovine fibronectin were prepared as previously described (Martin and Sutherland, 2001; Sutherland et al., 1993). Embryos were collected and cultured as described above and transferred to $10 \mu \mathrm{l}$ drops of medium overlaying fibronectin coated plastic at designated times and analyzed for trophoblast outgrowth at specific intervals.In experiments using inhibitors (Rapa, CHX, $\alpha$-amanitin, NO711, and BCH) embryos were first incubated for $1 \mathrm{~h}$ in $-\mathrm{AA}$ containing the specific inhibitor and then transferred to medium with amino acids and containing the same inhibitor. After 8 hours the embryos were washed three times in 550 $\mu \mathrm{l}$ of medium either containing or not containing amino acids depending on experimental protocol, and then transferred to fibronectin coated drops of medium and analyzed for trophoblast outgrowth at various intervals over the next 48 hours (Sutherland et al., 1993).

\section{Western Blotting}

Oocytes and 2-cell, 8-cell, morulae, and blastocyst stage embryos were collected over the course of several weeks and stored at $-80^{\circ} \mathrm{C}$ until needed, then prepared for immunoblotting by lysing in homogenization buffer ( $50 \mathrm{mM} \mathrm{NaF}, 1 \mathrm{mM}$ EDTA, 1mM EGTA, $1 \mathrm{mM}$ DTT, $10 \mathrm{mM}$ sodium phosphate, $50 \mathrm{mM} \beta$-glycerolphosphate $\mathrm{pH} 7.4,1 \mathrm{mM}$ PMSF, $10 \mu \mathrm{l} / \mathrm{ml}$ phosphatase inhibitor cocktail, $10 \mu \mathrm{g} / \mathrm{ml}$ proteinase inhibitor cocktail, $10 \mu \mathrm{g} / \mathrm{ml}$ DNAse, $1 \%$ Tween-20). After lysis, lysate equivalent to 100 oocytes or embryos was loaded per lane of a 4-12\% NuPage Novex Bis-Tris gradient gel (\#NP0323), run and then transferred to PVDF membrane (Milipore, Billerica, MA). The membrane was blocked with 5\% Non fat milk solution in TBS/T (Blocking Buffer) for 1 hour at room temperature, then incubated at $4^{\circ} \mathrm{C}$ overnight in buffer $(1 \times$ TBS with 5\%BSA, $0.01 \%$ Tween-20) containing primary antibody. After washing, the blot was incubated with HRP-conjugated donkey anti rabbit secondary antibodies in blocking buffer (BioLegend, San Diego, MA) for 1 hour at room temperature, washed again and then chemiluminescence (Pierce, Rockford, IL) was used to detect the proteins of interest; SLC6A14, p70S6K, 4EBP, mTOR, Rictor, Raptor, Lst8 and tubulin, as well as phosphorylated forms of p70S6K and 4EBP.

\section{Uptake of $\left[{ }^{3} \mathrm{H}\right]$ Arginine and $\left[{ }^{3} \mathrm{H}\right]$ leucine}

Embryos were cultured in various amino acid conditions for 8 hours from 120-128h phCG. At $128 \mathrm{~h}$ phCG, embryos were transferred to + AA medium containing $\left[{ }^{3} \mathrm{H}\right]$ arginine or $\left[{ }^{3} \mathrm{H}\right]$ leucine substituted for non-radiolabeled arginine or leucine to the same concentration. After 10 minutes incubation in $\left[{ }^{3} \mathrm{H}\right] \mathrm{AA}$ medium, embryos were washed three times in 550 $\mu \mathrm{l}$ of cold -AA, then placed into scintillation vials and lysed with $100 \mu \mathrm{l}$ boiling $2 \%$ SDS. Scintillation fluid (Ready Safe) was added, the vial was thoroughly vortexed, and the radioactivity of the sample measured using a liquid scintillation counter (Beckman LS1801). For inhibition experiments with $\mathrm{BCH}$ and NO711, embryos were first incubated with the inhibitor in -AA medium for one hour at $120 \mathrm{~h}$ phCG and then transferred for 8 hours into amino acid containing medium from $121-129 \mathrm{~h}$ phCG followed by a 10 minute pulse of [ ${ }^{3} \mathrm{H}$ ] amino acid pulse and liquid scintillation detection as described above. 


\section{Immunofluorescence}

Oocytes and embryos were fixed in $3.7 \%$ formaldehyde in immunofluorescence (IF) buffer (40mM PIPES pH 6.8, 200mM NaCl, 2mM EGTA) for 15 minutes, washed three times in IF buffer for 5 minutes, permeabilized with o.1\% Triton X-100/IF buffer for 2 minutes, washed three times in IF buffer for 5 minutes and blocked in 1 hour at room temperature in Super Block (5\%BSA, 20\%FBS). Oocytes or embryos were then incubated in Super Block with primary antibody added $(1: 200)$ overnight at $4{ }^{\circ} \mathrm{C}$. The next day they were washed three times in 0.5\% Tween20/IF Buffer for 5 minutes and placed in 0.5\% Tween20/IF Buffer solution with secondary antibody added (Invitrogen, Carlsbad, CA) for 1 hour at room temperature. Lastly, oocytes and embryos were washed three times in $0.5 \%$ Tween20/IF Buffer and 1X IF Buffer for five minutes, mounted in DAPI/PBS, and analyzed by confocal and multiphoton microscopy.

\section{Confocal Microscopy and Image Analysis}

The imaging system consisted of a Zeiss Axiovert 200M epifluorescent motorized microscope with a $100 \mathrm{~W} \mathrm{Hg}$ arc lamp and a halogen lamp. AC-Apochromat $63 \times$ water NA 1.2 objective lens was used for the studies described here. The Axiovert 200M was coupled to a Zeiss510 confocal-multiphoton-spectral imaging system (www.zeiss.de), and the system was controlled using the LSM software (version 4.0). For confocal imaging, the system consisted of a $45 \mathrm{~mW}$ argon laser $(458,488,514 \mathrm{~nm})$, a $10 \mathrm{~mW}(561 \mathrm{~nm})$ laser and a $633 \mathrm{~nm}$ laser. We used $561 \mathrm{~nm}$ to excite the Alexa546 fluorophore donkey anti rabbit secondary antibody. For two-photon imaging a 10W Verdi pumped, tunable (model 900 Mira, www.coherentinc.com) modelocked ultrafast ( $78 \mathrm{MHz}$ ) pulsed ( $<150$ femtosecond) laser was coupled to the laser port of a Zeiss510 META/NLO. This laser was equipped with optics for easy tunable range of the entire wavelength (700 to 1,000 nm). The system was equipped with spectrum analyzer (Model E201; www.istcorp.com) to monitor the excitation wavelength. We used two-photon $830 \mathrm{~nm}$ laser to excite the DAPI. Scanning confocal image stacks were taken at $0.5 \mathrm{micron}$ intervals. Confocal data was then analyzed using Volocity (Improvision, Waltham, MA) to render three-dimensional reconstructions and to capture images.

\section{Uterine fluid collection and spreading experiments}

Uterine fluid was collected using methods previously described (Harris et al., 2005) from 810 mice per stage at 48,60,72,120,130, and $144 \mathrm{~h}$ phCG. The fluid collected at each time point was pooled together, spun down, and stored at $-20^{\circ} \mathrm{C}$ under oil until just prior to use. In vitro matured $120 \mathrm{~h}$ phCG embryos were placed in $5 \mu 1$ drops of $-\mathrm{AA}$ medium on fibronectin substrates, then the -AA medium was removed and replaced with the warmed and $\mathrm{CO}$-equilibrated uterine fluid, and the embryos were then cultured and analyzed at specific times for trophoblast outgrowth.

\section{Statistical Analysis}

For experiments examining trophoblast outgrowth and amino acid uptake, the data from a minimum of three experiments done in triplicate were averaged and the standard deviation calculated. The averages and standard deviations were analyzed for statistical significance using one-tailed ANOVA and the Student-Newman-Keuls test (Glantz, 1992). 


\section{RESULTS}

\section{Arginine and leucine are necessary and sufficient for blastocyst activation and trophoblast outgrowth}

Previous results have shown that amino acids are required at the early blastocyst stage ( $120 \mathrm{~h}$ post-hCG for superovulated embryos, Table 1) for the embryo to initiate the motility required for implantation (Gwatkin, 1966; Martin and Sutherland, 2001; Martin et al., 2003; Naeslund, 1979). Early results suggested that leucine and arginine were particularly important for initiation of embryo outgrowth (Gwatkin, 1966; Naeslund, 1979). Excluding either arginine or leucine, or both, from the culture medium results in quiescent embryos that fail to form outgrowths when placed on a fibronectin substrate (Figure 1A) while exclusion of other individual AA does not affect trophoblast motility. Notably, this includes glutamine, which in previous work in cultured cells was found to be necessary for leucine uptake (Nicklin et al., 2009). These data confirm that the availability of arginine and leucine alone are most critical to the initiation of in vitro trophectoderm spreading.

Since arginine and leucine are required for trophoblast outgrowth, we tested whether these amino acids were sufficient to induce trophectoderm spreading. An 8-hour pulse of either arginine or leucine alone, at an optimal concentration of $0.2 \mathrm{mM}$, partially rescues spreading (Figure 1B). This effect is unique to either arginine- or leucine-containing medium, as medium containing other individual AAs fail to induce trophoblast outgrowth (Figure 1B). Notably, the effects of arginine and leucine are additive. When embryos are stimulated with $0.2 \mathrm{mM}$ arginine and leucine in combination for $8 \mathrm{~h}$, the number of embryos exhibiting trophoblast outgrowth is significantly higher, while continuous combined exposure leads to a complete rescue of trophoblast outgrowth to control levels. In contrast to results obtained in cultured cells (Nicklin et al., 2009), glutamine does not synergize with leucine, and is inhibitory to arginine (Figure 1B).Taken together, these data show that arginine and leucine each contribute individually to the induction of trophoblast outgrowth, and their effects are additive when in combination.

\section{Leucine transport by the amino acid transporter SLC6A14 (ATB ${ }^{\circ,+}$ ) is required for trophoblast outgrowth}

Embryos take up and release amino acids via specific transporters. Embryos express a large repertoire of transporters that vary in substrate preference, and importantly, change according to embryonic stage (Van Winkle, 2001; Van Winkle et al., 1985; Van Winkle et al., 2006). Leucine transport in particular, changes with the onset of the blastocyst stage, becoming predominantly dependent on the SLC6A14 transporter (also designated ATB ${ }^{0,+}$ ). SLC6A14 is a sodium-dependent, neutral and cationic amino acid transporter with a broad specificity but with a high affinity for leucine, isoleucine, and methionine (Borland and Tasca, 1974; Van Winkle et al., 1985; Van Winkle et al., 2006).

The activity of SLC6A14 is likely regulated at the level of transcription, as the message is not detected during preimplantation development until the blastocyst stage (Martin et al., 2003; Van Winkle et al., 1990a). Protein expression follows a similar pattern. Although 2cell embryos retain a robust amount of maternally inherited SLC6A14, it is rapidly degraded by the 8-cell stage (Figure 2A). Levels of SLC6A14 protein remain low at the morula stage but rapidly rise in the blastocyst, coincident with the onset of SLC6A14 gene transcription, and with the onset of competency to receive activation signals (Martin and Sutherland, 2001). Exposing embryos to AA further increases SLC6A14 expression, suggesting that its own translation may be affected by mTOR activity (Figure 2A).

To test whether leucine transport by SLC6A14 is central to trophoblast outgrowth, we used NO711, a non-substrate inhibitor of SLC6A14 (Sloan, 2002), and BCH, a competitive 
inhibitor of leucine transport that has been previously shown to inhibit leucine uptake by SLC6A14 (Sloan and Mager, 1999). NO711 treatment blocked leucine uptake (Figure 2D) and trophoblast outgrowth both in the presence of all AA and in the presence of leucine alone (Figure 2B and Supplemental Figure 1A). Embryos cultured in + Arg medium were unaffected by the addition of NO711, consistent with the fact that arginine uptake is predominantly through the Slc3a1/Slc7a9 $\left(\mathrm{b}^{0,+}\right)$ heterodimeric amino acid transporter (Van Winkle et al., 1990b). In +Leu/+Arg medium NO711 reduced spreading to levels comparable to those in medium containing arginine alone (Figure 2B). Treatment of embryos with BCH yielded similar results (Figure 2B).

We examined the specific requirement for SLC6A14 transport in two ways. First, we cultured embryos in medium containing NO711 and increasing levels of leucine, which resulted in greatly increased uptake of leucine through other transporters, but no increase in trophoblast outgrowth (Figure 3A, B). Secondly, SLC6A14 also transports methionine and isoleucine with similar $\mathrm{K}_{\mathrm{m}} / \mathrm{K}_{\mathrm{i}}$ to leucine (Sloan and Mager, 1999; Van Winkle et al., 1985; Van Winkle et al., 1988; Van Winkle et al., 2006). However, embryos cultured in medium containing arginine in combination with either methionine or isoleucine show no additive effect on trophoblast outgrowth (Supplemental Figure 1B). These data show that leucine transport specifically via SLC6A14 is critical for stimulation of trophoblast outgrowth as well as for the combined action of arginine and leucine together.

\section{Glutamine blocks the effects of arginine on trophoblast outgrowth}

Eliminating glutamine from embryo culture does not affect TGC outgrowth, nor does medium solely containing glutamine induce spreading (Figure 1A,B). Interestingly, when embryos are cultured in the presence of $0.2 \mathrm{mM}$ arginine and $4.0 \mathrm{mM}$ glutamine, arginine uptake and trophoblast outgrowth is completely inhibited (Figure 1B). Conversely glutamine does not inhibit spreading either in complete amino acid conditions or in medium containing a combination of leucine and glutamine (Figure 1B). Glutamine's negative effects are exclusive to arginine, and may explain why embryos fail to spread when leucine is individually excluded from the culture medium despite the continued presence of arginine (Figure 1A).

\section{Arginine and leucine rescue is rapamycin sensitive}

We previously showed that AA induced trophoblast outgrowth is a rapamycin sensitive event, and thus is upstream of the serine/threonine kinase complex mTORC1. Rapamycin is an immunophillic macrolide that allosterically inhibits mTORC1 activity by specifically binding to the protein mTOR when complexed with Raptor and LST8 in the mTORC1 complex. Given that arginine and leucine are individually required for trophoblast outgrowth, we hypothesized that each had a separate, required, action that together led to blastocyst activation, and that one of these separate actions was mTOR activation. We tested this hypothesis by culturing embryos in medium containing either arginine or leucine alone or both in combination, and adding rapamycin to block mTORC1 activity. Rapamycin completely blocked trophoblast outgrowth under all three conditions (Figure 4A). Thus the effects of both arginine and leucine are rapamycin-sensitive, implying that each activates mTORC1.

\section{Arginine and leucine induction of trophoblast outgrowth is dependent upon translation but independent of transcription}

mTORC1 activates translation of specific populations of mRNAs, including TOP RNA and cap dependent translation of mRNAs containing particularly complicated 5' untranslated regions (De Benedetti and Rhoads, 1990; Koromilas et al., 1992; Lazaris-Karatzas et al., 1990). Induction of trophoblast outgrowth by +AA medium requires translation (Martin and 
Sutherland, 2001), so we hypothesized that the effect of arginine and/or leucine on trophoblast outgrowth is similarly dependent upon translation. To test this, embryos were cultured for 8 hours in medium known to promote spreading (+AA, +Arg, +Leu, and +Arg/ Leu) and cycloheximide (CHX) was added at concentrations known to inhibit mRNA translation (Epstein and Smith, 1973). The presence of CHX inhibited trophoblast outgrowth in all experimental groups (Figure 4B). These results indicate that the effect of both arginine and leucine on trophoblast outgrowth requires protein translation. We next tested whether transcription was necessary for the induction of trophoblast spreading. Embryos were cultured for 8 hours in medium known to promote spreading (+AA, +Arg, +Leu, and +Arg/ Leu) and $\alpha$-amanitin was added at concentrations known to inhibit mRNA transcription (Flach et al., 1982; Levey and Brinster, 1978; Lindell et al., 1970). The presence of $\alpha$ amanitin did not affect spreading, indicating that the effects of arginine and leucine are independent of transcription during the 8 hours of AA exposure (Figure 4C).

\section{Phosphorylation of known downstream targets of mTORC1 fails to correlate with embryo spreading}

mTORC1's effects on translation are accomplished via the phosphorylation of two well established downstream effectors, p70S6K and 4EBP. p70S6K and 4EBP both regulate protein translation (Lin et al., 1994; Pause et al., 1994). Phosphorylation by mTORC1 at Thr389 activates p70S6K, which in turn phosphorylates ribosomal protein S6, and may influence translation of TOP RNAs (De Benedetti and Rhoads, 1990; Koromilas et al., 1992; Lazaris-Karatzas et al., 1990; Shama and Meyuhas, 1996) . Phosphorylation of 4EBP by mTORC1 relieves its repression of eIF4E, and promotes cap dependant translation by increasing the availability of eIF4E (Brunn et al., 1997; Pause et al., 1994). We next sought to determine whether the phosphorylation states of p70S6K and 4EBP correlated with embryo spreading. As expected, embryos stimulated with +AA medium exhibited a strong amount of p70S6K phosphorylation at Thr389, which is decreased in embryos cultured in -AA medium or in +AA medium containing rapamycin (Figure 5A,C). Under all other culture conditions tested, phosphorylation of p70S6K was low. Under conditions where trophoblast outgrowth is stimulated (+Arg, +Leu, and +Arg/+Leu) p70S6K was phosphorylated to a similar degree as in -AA culture conditions (Figure 5C), while under conditions lacking leucine or both arginine and leucine p70S6K was phosphorylated to an even lower level (Figure 5A,C).Surprisingly however, p70S6K phosphorylation was fairly robust in embryos cultured in medium lacking arginine, where embryos did not spread at all (Figure 5 A,C).

mTORC1 phosphorylates 4EBP in a hierarchical fashion, first at Thr36/45 (Thr37/46 in mouse) and Thr69, and then at Ser64 (Ser65 in mouse) to fully relieve its association with eIF4E (Fadden et al., 1997; Gingras et al., 1999; Mothe-Satney et al., 2000b), and phosphorylation of Thr69 and Ser64 are more sensitive to rapamycin (Mothe-Satney et al., 2000a). 4EBP is phosphorylated both on Thr37/46 and Ser65 in embryos cultured in +AA medium, and phosphorylation of both sites is decreased in embryos cultured in - AA medium or in +AA medium containing rapamycin, with greater decreases in phosphorylation seen for Ser65 in the presence of rapamycin, as expected (Figure $5 \mathrm{~A}, \mathrm{~B}$, C). Overall, though, the phosphorylation of $4 \mathrm{EBP}$ at both sites appears to be inversely correlated with the ability of the embryo to initiate trophoblast outgrowth. Under conditions where trophoblast outgrowth is partially rescued (+Arg, +Leu) 4EBP is only moderately phosphorylated both on Thr37/46 and Ser65, with somewhat more robust phosphorylation in the +Arg/+Leu combination group (Figure $5 \mathrm{~A}, \mathrm{~B}, \mathrm{C}$ ). However, 4EBP is strongly phosphorylated at both Thr37/46 and Ser65 in embryos cultured under conditions where trophoblast outgrowth is inhibited (-Arg, -Leu and - Arg/-Leu) (Figure 5 A, B,C). These data indicate that despite the fact that arginine and/or leucine initiate a signal cascade that 
requires both $\mathrm{mTORC} 1$ and protein translation in order to produce changes in cell behavior and motility, the activities of the well established downstream targets of mTORC1 that mediate its effects on translation fail to correlate with the ability of the embryo to initiate trophoblast outgrowth.

\section{The amino acid sensitive mTORC1 components are preferentially expressed in the mouse blastocyst}

Since mTOR can form two functionally distinct complexes we next examined the developmental profile of the various protein components of mTORC1 and mTORC2 to provide insight into the relevance of these two complexes in relation to each other and with respect to implantation. mTOR protein levels remain steady from oocyte to blastocyst while Raptor protein levels specifically increase in morulae and blastocysts (Figure 6). Conversely, levels of the mTORC2-specific subunit Rictor are relatively high in the oocyte and 2-cell embryo, but diminished in the blastocyst (Figure 6). Interestingly, protein expression of the common subunit LST8 also decreases in the blastocyst (Figure 6). It appears that mTOR and Raptor are preferentially expressed in the implantation stage embryo while Rictor and LST8 are not. These data are consistent with the results of mouse knockouts (Gangloff et al., 2004; Guertin et al., 2006). Knockouts of mTOR and Raptor are lethal at the peri-implantation stage, while knockouts of Rictor and LST8 are able to implant normally and develop to mid-gestation. These findings suggest that mTOR and Raptor functions are most critical to the implanting embryo and that the embryonic rapamycinsensitive mTORC1 is unique in that it may operate or exist without LST8.

\section{mTOR localization changes throughout development and upon embryo activation}

Although mTOR protein levels remain steady from oocyte to blastocyst, its cellular distribution changes substantially during this period (Figure 6). Oocytes and embryos at 48 and 68h phCG (2-cell and 8-cell respectively, Table 1) exhibit diffuse cytoplasmic localization of mTOR. As the embryo reaches $72 \mathrm{~h}$ phCG (morula, Table 1) mTOR localization begins to change from a diffuse distribution into aggregates of perinuclear puncta (Figure 7). The $120 \mathrm{~h}$ phCG embryo (early blastocyst, Table 1) retains this change in localization with distinct perinuclear puncta and additional plasma membrane localization (Figure 7). Stimulation of embryos with medium containing all AA results in a large increase in immunofluorescent staining intensity (Figure 8B.). Embryos cultured in +AA medium containing rapamycin (+AA Rapa) show an intermediate phenotype, with a modest amount of increased mTOR signal (Figure 8C), as well as a loss of the plasma membrane localization. The change in immunofluorescent signal intensity following AA exposure is antibody-specific, and is consistently seen when using the rabbit monoclonal antibody 7C10 from Cell Signaling Technology. This antibody recognizes a region of the protein at the Cterminus around the autophosphorylation site at Ser2481, phosphorylation of which is the major indicator of mTOR activation (Yang and Guan, 2007). We verified that the change in fluorescent intensity is not due to an overall change in protein expression in two ways. First we examined protein expression directly by Western blot of whole cell lysate from embryos cultured in either +AA or -AA, and found no difference in protein levels (Fig 8J). Second, we performed double-label immunofluorescence using the Cell Signaling Technology antimTOR antibody and a mouse monoclonal antibody from Santa Cruz Biotechnology (\#sc-136269) that was raised against amino acids 185-290 from the N-terminus of mTOR. The localization of mTOR using the Santa Cruz antibody was similar to that seen with the Cell Signaling antibody, but its staining intensity was similar in embryos cultured in -AA and those cultured in +AA (Fig S3). Collectively these data suggest that the increase in fluorescence seen when using the Cell Signaling Technology antibody may be due either to a change in conformation of mTOR, to phosphorylation of Ser2481, or to an unmasking 
event following mTOR activation, any of which might allow the antibody to more efficiently recognize its epitope.

Regardless of the underlying mechanism, the dramatic change in mTOR signal underscores the effect of amino acids on mTORC1 activation, and also serves as a powerful tool to monitor the activation state of mTOR directly in the embryo. Consistent with outgrowth data, embryos cultured either in +Arg, +Leu, or +Arg+Leu medium exhibited an activated pattern of mTOR immunostaining (Figure 9). Embryos cultured in -Leu, -Arg, or -ArgLeu medium also all exhibited an activated pattern of mTOR immunostaining (Figure 9). This is consistent with the Western blot data showing phosphorylation of mTORC1 target proteins, and confirms that mTORC1 activity is necessary but not sufficient to induce blastocyst activation.

\section{Embryos receive activation signals in vivo}

Trophoblast outgrowth is an in vitro manifestation of the invasive behavior that the trophoblast cells exhibit as they initiate implantation. Because the in vitro conditions under which embryos are cultured are different than those that they encounter within the uterus, we examined the dynamics of mTOR activation in embryos developing within the uterus. Mice were superovulated and mated as before, but the embryos were then flushed from the uteri at 96, 120, and 130h phCG (Table 1), cultured for 8 hours in medium either containing AA or not, and then transferred to a fibronectin substrate in medium lacking AA to assay the induction of trophoblast outgrowth. Embryos flushed at $96 \mathrm{~h}$ phCG embryos did not form outgrowths after $8 \mathrm{~h}$ culture in either medium, indicating that at this stage the embryo is not yet competent to become activated, and also has not yet been activated in vivo (Figure 10A). Embryos flushed at $120 \mathrm{~h}$ post-hCG did spread following culture in +AA medium, but did not when cultured in -AA medium (Figure 10A). Moreover mTOR staining of embryos flushed at $120 \mathrm{~h}$ phCG resembled that of in vitro cultured morulae and blastocysts prior to AA exposure (Figure 10B, C). In contrast, embryos flushed at 130h phCG were able to spread after culture either with or without AA, and mTOR staining of these embryos resembled that of in vitro cultured embryos following exposure to AA, when mTOR has been activated (Figure 10A,B,E). These data indicate that in vivo, the embryo becomes competent by $120 \mathrm{~h}$ phCG to respond to environmental conditions by activating mTOR, and further, that during the period between $120 \mathrm{~h}$ phCG to $130 \mathrm{~h}$ phCG changes occur in the uterine milieu that provide the blastocyst with signals that lead to mTOR activation and induction of trophoblast motility.

\section{In vitro derived blastocysts can be activated by uterine fluid isolated at $130 \mathrm{~h}$ phCG}

To verify that uterine fluid became competent to activate trophoblast motility by $130 \mathrm{~h}$ phCG, we collected uterine fluid from mice at various stages of pregnancy: 48, 60, 96, 120, $130,142,154 \mathrm{~h}$ phCG, and then used it to stimulate in vitro matured $120 \mathrm{~h}$ phCG embryos (early blastocysts), the peri-implantation stage known to be competent to receive activation signals. Embryos cultured in uterine fluid isolated 48-120h phCG fail to become activated, and exhibit no spreading on fibronectin substrates (Figure 11). However, uterine fluid isolated at $130 \mathrm{~h}$ phCG was able to promote trophoblast outgrowth, indicating that it contained in vivo derived elements capable of activating $120 \mathrm{~h}$ phCG embryos (Figure 11). Interestingly, fluid collected at $144 \mathrm{~h}$ phCG was not able to induce trophoblast outgrowth. These results confirm that the uterine environment changes very specifically between 120 and $130 \mathrm{~h}$ phCG to provide a signal to the embryo stimulating the onset of trophoblast motility. 


\section{Discussion}

Implantation is a central event in mammalian reproduction and is therefore a tightly regulated and coordinated event. Two independent but concomitant events, uterine receptivity and embryonic development, must be synchronized in order to ensure efficient implantation, and thus achieve maximal reproductive success. To enable this synchronization, an intricate language has evolved allowing the embryo and the uterus to be in constant communication. The nature of the signals underlying this communication is not well understood, but both previous work and the results presented here demonstrate that amino acid activation of mTORC1 is an important aspect of blastocyst activation, in particular for induction of motile, invasive behavior in the trophectoderm (Martin and Sutherland, 2001; Martin et al., 2003).

In this paper we demonstrate that of all the amino acids tested, only arginine and leucine are absolutely required for blastocyst activation. When either arginine or leucine, or both, are excluded from the culture medium, trophoblast outgrowth on fibronectin, a behavior indicative of blastocyst activation, is completely inhibited. In addition, arginine and leucine together are also completely sufficient to induce trophoblast outgrowth. Individually, either arginine or leucine partially induces blastocyst activation, and together they act additively to fully induce embryo activation. Interestingly, very similar results have been described for ovine conceptuses, where leucine and arginine, but not glutamine, are able to promote proliferation and trophoblast motility (Kim et al., 2011). Our findings differ somewhat from those reported by Van Winkle et al who found that arginine produced only a small amount of activation (Van Winkle et al., 2006). This discrepancy can most likely be attributed to differences in amino acid concentration $(0.2 \mathrm{mM}$ here vs $0.01 \mathrm{mM}$ in that study), and perhaps to differences in the embryonic state of development. While we used in vitro matured blastocysts cultured from 40h post-hCG in a simple defined medium, Van Winkle et al. used in vivo matured, delay of implantation embryos on day 8 of pregnancy, which may have been exposed to uterine factors that could change their behavior.

Our results confirm early observations that the mouse embryo requires arginine and leucine (Gwatkin, 1966; Martin and Sutherland, 2001; Naeslund, 1979), but importantly they dissect out the purpose, parameters, and proteins central to this requirement. Our results suggest that arginine and leucine have overlapping functions in stimulating trophoblast motility as demonstrated by their mutual requirement for mTORC1 activity for trophoblast outgrowth. However, we also demonstrate that arginine and leucine have independent, non-overlapping functions that are additive in stimulating blastocyst activation. It is possible that this 'two key lock' opened by arginine and leucine serves either as a dual developmental checkpoint or else as a fine-tuning mechanism for the onset of implantation.

Embryos express a repertoire of amino acid transporters that can change according to developmental stage. SLC6A14 is a Na$/ \mathrm{Cl}^{-}$dependant transporter capable of forming AA gradients (Borland and Tasca, 1974; Van Winkle et al., 1985; Van Winkle et al., 2006). Protein expression of SLC6A14 is specifically upregulated at the blastocyst stage, coincident with the onset of competence to receive an extrinsic activation signal, and further increases upon AA exposure. SLC6A14 plays an important role in blastocyst activation. It is the major transporter for leucine in the blastocyst (Van Winkle et al., 1990a), and significantly, leucine in particular must be transported via SLC6A14 to contribute to embryo spreading. Inhibiting leucine uptake through SLC6A14 by treatment with either NO711 or $\mathrm{BCH}$ leads to inhibition of trophoblast outgrowth, which cannot be rescued by increased leucine uptake through other transporters. Other high-affinity substrates for SLC6A14 such as isoleucine or methionine (Sloan and Mager, 1999; Van Winkle et al., 1985; Van Winkle et al., 1988; Van Winkle et al., 2006) cannot substitute for leucine, indicating that there is a 
role both for the amino acid and for the action of the transporter. Since SLC6A14 is an active transporter, it can both concentrate amino acids within the cell and can simultaneously create a charge gradient by co-transport of $2 \mathrm{Na}+$ and $1 \mathrm{Cl}$ - ion (Sloan and Mager, 1999). The concentrative effect was hypothesized to be important in stimulating uptake of arginine through SLC3A1/SLC7A9, a heterodimeric transporter which functions as a tightly coupled amino acid antiporter (Martin et al., 2003; Palacin et al., 2005; Van Winkle, 2001). Our results, however, do not support this hypothesis, as arginine uptake is not stimulated by addition of leucine, nor is it inhibited either when leucine is not present in the medium or when leucine uptake through SLC6A14 is inhibited. Furthermore, the concentration of leucine within the cell does not appear to be the limiting factor, since uptake of leucine at normal levels through other transporters is not sufficient to induce trophoblast outgrowth. This suggests that either the changes in intracellular concentration of $\mathrm{Na}$ or $\mathrm{Cl}$, or any resulting changes to the membrane potential may synergize with the intracellular effects of leucine to induce blastocyst activation.

The timely expression of SLC6A14 at the blastocyst stage serves as a key regulator of blastocyst activation, bestowing upon the $120 \mathrm{~h}$ phCG embryo the ability to receive an activation signal both in vitro and in vivo. In addition to the embryo's temporal regulation of SLC6A14, factors related to the onset of uterine receptivity may also act to regulate its activity in the embryo. For example, a global change in the concentration of leucine in the uterine milieu could drive uptake and subsequent embryo activation. Alternatively, concentrations of $\mathrm{Na}+$ in the uterus have been found to increase prior to implantation (Nilsson and Ljung, 1985; Van Winkle et al., 1983) and since SLC6A14 is a Na+ dependant transporter, an overall increase in $\mathrm{Na}+$ concentration could directly affect transport activity. Such an idea is further supported by the finding that blastocysts do not form outgrowths when $\mathrm{Na}+$ levels are reduced in culture conditions but become activated with increased $\mathrm{Na}+$ concentration (Van Winkle, 1981). These and other scenarios are equally plausible. Determination of uterine fluid composition at various hours phCG is needed to address this point and future work will address these possibilities.

It is well documented that AAs, in particular arginine and leucine, activate the serine/ threonine protein kinase mTOR. mTOR can form two biochemically and functionally distinct complexes, the traditionally rapamycin sensitive mTORC1 and insensitive mTORC2. While levels of the mTORC2 subunit Rictor are almost undetectable in the blastocyst, the mTORC1 core component Raptor is preferentially expressed at this stage. Interestingly, levels of the common component LST8 decrease from oocyte throughout early preimplantation stages, and are nearly undetectable in the blastocyst. The expression data support the predominant role for mTORC1 in implantation established in mouse knockouts for mTOR, Rictor, Raptor, and LST8 (Gangloff et al., 2004; Guertin et al., 2006). Somewhat different results have been described for ovine embryos, where arginine treatment leads to increased Akt1 phosphorylation, suggestive of mTORC2 activation (Kim et al., 2011).

In the presence of rapamycin, +AA medium fails to stimulate trophoblast outgrowth, implicating mTORC1 activity in regulating this behavior (Gangloff et al., 2004; Martin and Sutherland, 2001). Likewise embryos cultured in +Arg, +Leu, or +Arg+Leu medium in the presence of rapamycin, fail to exhibit trophoblast outgrowth, placing arginine and leucine, both individually and in combination, upstream of mTORC1 in the pathway inducing trophoblast motility. A major function of mTORC1 is to regulate cellular growth by phosphorylation of two downstream effectors, p70S6K and 4EBP, which in turn increase the translation of specific populations of mRNAs (De Benedetti and Rhoads, 1990; Koromilas et al., 1992; Lazaris-Karatzas et al., 1990; Lin et al., 1994; Pause et al., 1994; Shama and Meyuhas, 1996). The effects of either + Arg or + Leu medium on trophoblast motility require protein translation but not mRNA transcription during the 8-hour pulse of amino acid 
treatment. These observations suggest that novel proteins are required to promote the changes in trophectoderm cell motility required for implantation, but that these proteins are unlikely to be transcription factors that induce new gene expression.

Surprisingly, despite the clear requirement for mTORC1 activity and new translation, trophoblast outgrowth was not correlated with the activity of either p70S6K or 4EBP, as measured by their level of phosphorylation by mTORC1. While only moderate levels of phosphorylation of p70S6K and 4EBP were seen in embryos cultured under conditions that promote trophoblast outgrowth ( $+\mathrm{Arg},+\mathrm{Leu},+\mathrm{Arg}+\mathrm{Leu}$ ), we detected robust amounts of phosphorylation of both proteins in embryos cultured under conditions where trophoblast outgrowth is inhibited (-Arg, -Leu, -Arg-Leu). These observations suggest the presence of novel rapamycin-sensitive actions of mTORC1 independent of both p70S6K and 4EBP1. This possibility seems quite plausible given that mTOR affects a wide variety of signaling pathways and cellular processes (Barbet et al., 1996; Di Como and Arndt, 1996; Kunz et al., 1993; Laplante and Sabatini, 2009a, b). Alternatively, a rapamycin-sensitive mTOR complex lacking LST8 (mTORC3?) might have alternative downstream targets in the blastocyst (Fig 12). Only low levels of LST8 are detected in the blastocyst, and embryos lacking LST8 do implant (Guertin et al., 2006).

mTORC1 has also been shown to play a central role in skeletal myogenesis, independent of kinase activity and the function of p70S6K and 4EBP (Erbay and Chen, 2001). In this system mTOR regulates expression of IGF-II through both transcriptional and posttranscriptional mechanisms (Erbay et al., 2003; Ge et al., 2011). IGF-II is an interesting candidate as a potential regulator of trophoblast differentiation in that it is preferentially expressed in TE cells at the time of implantation (Lee et al., 1990), and is known to promote trophoblast cell differentiation (Kanai-Azuma et al., 1993). However, the mechanisms of regulation would likely be different in trophoblast cells from those defined during myogenesis, given that de novo transcription is not necessary for trophoblast outgrowth.

Although mTORC1 has primarily been studied in relation to its effects on cellular growth and proliferation, effects on motility are not entirely unprecedented. Activation of mTORC1 specifically in keratinocytes leads to accelerated wound healing both in vitro and in vivo (Squarize et al., 2010) although the precise nature of the pathways responsible for this behavioral transformation remain elusive. Actin cytoskeletal dynamics are likely involved in the observed changes in trophoblast cell motility, and although mTORC2 activity is traditionally thought to affect the cytoskeleton, it remains equally plausible that mTORC1 may also exert significant effects on actin dynamics. Not only does mTORC1 interact with Rho GTPases, such as P-Rex1, (Hernandez-Negrete et al., 2007) it also plays a role in the progression and metastasis of colorectal cancer through RhoA and Rac1 activation (Gulhati et al., 2011). Given the similar cell behaviors during the epithelial to mesenchymal transition that underlies both metastasis and implantation, it seems possible that activated embryonic mTORC1 may act in analogous manner to drive trophoblast motility.

While our results provide strong evidence that an activated, rapamycin sensitive mTOR complex is necessary for blastocyst activation, they also indicate that an activated mTOR complex is not sufficient. While mTOR is active in embryos cultured in -Arg or -Leu medium as assessed both by increased p70S6K and 4EBP phosphorylation and by immunostaining of mTOR (Figs ), these embryos remain unable to form trophoblast outgrowths. This suggests that while arginine and leucine activate a rapamycin sensitive mTOR complex that in turn activates translation, these two amino acids also have independent effects either downstream of or parallel to mTOR signals (Fig 12). Studies of ovine embryo development suggest a role for polyamines and nitric oxide downstream of arginine (Kim et al., 2011). Expression of ornithine decarboxylase, a rate-limiting enzyme in 
the production of polyamines from arginine, is highly associated with implantation in the mouse although inhibition by either chemical (Zhao et al., 2008) or genetic (Pendeville et al., 2001) approaches does not prevent implantation. IGF-II induces production of the arginine derived bioactive molecule nitric oxide (NO) (Kaliman et al., 1999; Lee et al., 1990) which can promote cell motility by modifying cell substrate adhesion (Brown et al., 2001; Chang et al., 2002; Kaur et al., 1998; Liu et al., 2006). Importantly, disruption of NO signaling inhibits formation of mouse blastocyst trophoblast outgrowths (Sengoku et al., 2001). Clearly, further characterization of arginine- and leucine-specific signals will be important to clarify the pathways regulating motility.

mTOR protein levels remain steady throughout preimplantation development, but its intracellular localization changes, which may reflect its functional status. Studies in cultured cell models have shown that amino acids promote activation of Rag GTPases and the trimeric Ragulator complex, which in turn localize mTOR to the surface of lysosomes where it can interact with its activator, Rheb cPase (Flinn et al., 2010; Sancak et al., 2010; Sancak et al., 2008). Through the 8-cell stage, embryos exhibit a diffuse cytoplasmic staining while morulae and blastocysts show an increasingly punctate perinuclear and plasma membraneassociated pattern. Embryos treated with + AA showed a substantial increase in mTOR signal despite no change in total mTOR protein expression, suggesting that amino acid signals lead either to a change in mTOR conformation, or to a change in association with other proteins that mask the antigenic epitope. In contrast, no appreciable change in Raptor signal intensity was observed. Rapamycin treatment does abrogate the increase in mTOR signal, although there is a modest signal boost. The change in mTOR immunofluorescent signal occurs very rapidly, within even 1' of +AA exposure, and remains elevated in embryos subsequently transferred to -AA medium (data not shown). The change in mTOR immunofluorescent intensity provides a very powerful tool for monitoring its activation state in intact embryos, and strongly supports the concept that mTOR activation is a checkpoint in blastocyst progression to the activated state. Embryos cultured in -AA medium in vitro remain in a quiescent, non-spreading state, but can be induced to spread with exposure to AA at any time after 120h phCG (Martin and Sutherland, 2001). The exposure to AA is an irreversible signal after 4-8 hours, as transfer back to -AA medium does not inhibit the ability of the embryo to initiate trophoblast outgrowth (Martin and Sutherland, 2001). Importantly, the difference in timing between mTOR activation ( $\leq 1$ minute) and the irreversible induction of trophoblast outgrowth (4-8 hours) also underscores our conclusion that mTOR activation is necessary but not sufficient for this process.

The mTOR activation checkpoint seen in vitro also occurs in vivo, and exhibits identical temporal dynamics. Embryos matured in vivo to $96 \mathrm{~h}$ phCG were unable to form trophoblast outgrowths following an 8-hour incubation in +AA, similar to in vitro matured embryos (Martin and Sutherland, 2001). Embryos matured in vivo to $120 \mathrm{~h}$ phCG exhibited a nonactivated pattern of mTOR localization and did not spread in the absence of exogenously supplied AA, but did form trophoblast outgrowths when cultured in +AA medium, again similar to those matured in vitro. This indicates that although the embryos are competent to receive an activation signal at $120 \mathrm{~h}$ phCG, it has not yet occurred in vivo. Conversely, by 130h phCG embryos not only exhibited increased mTOR signal intensity but spread in both $+\mathrm{AA}$ and -AA medium, indicating that an activation signal has occurred in vivo by this time. These results reveal the in vivo timeline of embryo activation and provide evidence for a specific change in the uterine milieu that is important for blastocyst activation. Further evidence supporting a specific, temporally regulated change in the uterine milieu was obtained by isolating uterine fluid from mice at the same stages of pregnancy. While uterine fluid from mice at 48-72 h phCG not only failed to produce trophoblast outgrowth, it also caused the embryos to bleb and appeared apoptotic. While fluid from $120 \mathrm{~h}$ phCG uteri was not toxic to blastocysts it did not stimulate trophoblast outgrowth. Significantly, uterine fluid 
from mice at $130 \mathrm{~h}$ phCG promoted trophoblast outgrowth by nearly all blastocysts, but uterine fluid from mice at $144 \mathrm{~h}$ phCG was not able to induce trophoblast outgrowth. Thus, as embryos progress from morning to evening on E4.5, uterine changes occur within a narrow window of time that induce mTOR activation and the onset of trophoblast motility in preparation for the initiation of invasion. These uterine changes could include modification either in amino acid composition, or in the concentration of $\mathrm{NaCl}$, which could in turn drive increased amino acid uptake. The timing of these changes suggests that they could be a downstream effect of LIF action on the decidualizing stroma and/or on the uterine epithelium (Ramathal et al., 2010). Alternatively, interaction of the embryo with uterinederived soluble signals, such as Wnt4 (Franco et al., 2011) or with HB-EGF on the uterine epithelium (Das et al., 1994a) may lead to intracellular signals regulating the localization or activity of SLC6A14. In particular, HG-EGF has been shown to induce calcium influx leading to accelerated trophoblast differentiation (Wang et al., 2000), and the SLC6A14 transporter has two consensus sites for phosphorylation by Protein Kinase C (PKC) and one for phosphorylation by CamKII which may affect its function (Sloan and Mager, 1999). In this way, attachment of the embryo to the uterine epithelium could conceivably facilitate transduction of the signal to initiate invasion.

Our data suggest that activation of trophoblast motility is independent of the process of apposition and attachment. Previous studies have identified a uterine signal required for initiation of apposition and attachment of the embryos to the uterine epithelium, which occurs in the late afternoon of E3.5, and leads to the onset of attachment by midnight of that evening (E4.0) (Das et al., 1994b; Paria et al., 1993a; Song et al., 2000). In vitro, blastocysts exhibit increased integrin trafficking to the plasma membrane and subsequently acquire the ability to become adhesive as exhibited by binding to fibronectin beads (Schultz and Armant, 1995; Schultz et al., 1997; Wang et al., 2002; Yelian et al., 1995). These changes in integrin distribution and fibronectin binding occur even in the absence of an AA signal (Martin and Sutherland, 2001), illustrating that the lack of trophoblast spreading in -AA conditions does not arise from an inability to become adhesive, but rather from an inability to initiate motility. Consistent with the in vitro results, we show here that the onset of motility is not induced in vivo until at least 10 hours after the onset of attachment, suggesting a two-step process by which the uterus signals to the embryo to promote implantation. Our results suggest that the second signal may result in changes in uptake of amino acids by the embryos, particularly of leucine and arginine, which in turn both activate mTORC1 and act independently and additively to induce trophoblast motility.

\section{Highlights}

Arginine and leucine are necessary and sufficient to induce trophoblast motility. Arginine and leucine have both mTORC1-dependent and independent actions. p70S6K or 4EBP activity do not correlate with motility suggesting a separate pathway. In vivo mTOR activation suggests uterine factors induce mTORC1 regulated motility. Amino acids may be components of the in vivo uterine-embryo implantation crosstalk.

\section{Supplementary Material}

Refer to Web version on PubMed Central for supplementary material.

\section{Acknowledgments}

We thank the members of the Sutherland lab for helpful discussions, and Drs. David Castle, James Casanova and Barry Hinton for generous gifts of reagents and very helpful discussions. This study was supported by a grant from the National Institutes of Health (HD034807) to A.E.S. I.M.G. was also supported by a training grant from the National Institute of General Medical Sciences (T32 GM08136). 


\section{BIBLIOGRAPHY}

Barbet NC, Schneider U, Helliwell SB, Stansfield I, Tuite MF, Hall MN. TOR controls translation initiation and early G1 progression in yeast. Molecular biology of the cell. 1996; 7:25-42.

[PubMed: 8741837]

Belchetz PE, Plant TM, Nakai Y, Keogh EJ, Knobil E. Hypophysial responses to continuous and intermittent delivery of hypopthalamic gonadotropin-releasing hormone. Science (New York, N.Y.). 1978; 202:631-633.

Bhatt H, Brunet LJ, Stewart CL. Uterine expression of leukemia inhibitory factor coincides with the onset of blastocyst implantation. Proceedings of the National Academy of Sciences of the United States of America. 1991; 88:11408-11412. [PubMed: 1722331]

Biggers JD, Borland RM, Powers RD. Transport mechanisms in the preimplantation mammalian embryo. Ciba Foundation symposium. 1977:129-153. [PubMed: 145938]

Bliss SP, Navratil AM, Xie J, Roberson MS. GnRH signaling, the gonadotrope and endocrine control of fertility. Frontiers in neuroendocrinology. 2010; 31:322-340. [PubMed: 20451543]

Borland RM, Tasca RJ. Activation of a Na+-dependent amino acid transport system in preimplantation mouse embryos. Developmental biology. 1974; 36:169-182. [PubMed: 4822831]

Brown C, Lin Y, Hassid A. Requirement of protein tyrosine phosphatase SHP2 for NO-stimulated vascular smooth muscle cell motility. American journal of physiology. Heart and circulatory physiology. 2001; 281:H1598-H1605. [PubMed: 11557549]

Brunn GJ, Hudson CC, Sekulic A, Williams JM, Hosoi H, Houghton PJ, Lawrence JC Jr, Abraham RT. Phosphorylation of the translational repressor PHAS-I by the mammalian target of rapamycin. Science (New York, N.Y.). 1997; 277:99-101.

Carson DD, Tang JP, Julian J. Heparan sulfate proteoglycan (perlecan) expression by mouse embryos during acquisition of attachment competence. Developmental biology. 1993; 155:97-106. [PubMed: 8416848]

Chang Y, Ceacareanu B, Dixit M, Sreejayan N, Hassid A. Nitric oxide-induced motility in aortic smooth muscle cells: role of protein tyrosine phosphatase SHP-2 and GTP-binding protein Rho. Circulation research. 2002; 91:390-397. [PubMed: 12215487]

Chen JR, Cheng JG, Shatzer T, Sewell L, Hernandez L, Stewart CL. Leukemia inhibitory factor can substitute for nidatory estrogen and is essential to inducing a receptive uterus for implantation but is not essential for subsequent embryogenesis. Endocrinology. 2000; 141:4365-4372. [PubMed: 11108244]

Das SK, Wang XN, Paria BC, Damm D, Abraham JA, Klagsbrun M, Andrews GK, Dey SK. Heparinbinding EGF-like growth factor gene is induced in the mouse uterus temporally by the blastocyst solely at the site of its apposition: a possible ligand for interaction with blastocyst EGF-receptor in implantation. Development. 1994a; 120:1071-1083. [PubMed: 8026321]

Das SK, Wang XN, Paria BC, Damm D, Abraham JA, Klagsbrun M, Andrews GK, Dey SK. Heparinbinding EGF-like growth factor gene is induced in the mouse uterus temporally by the blastocyst solely at the site of its apposition: a possible ligand for interaction with blastocyst EGF-receptor in implantation. Development (Cambridge, England). 1994b; 120:1071-1083.

De Benedetti A, Rhoads RE. Overexpression of eukaryotic protein synthesis initiation factor 4E in HeLa cells results in aberrant growth and morphology. Proceedings of the National Academy of Sciences of the United States of America. 1990; 87:8212-8216. [PubMed: 2122455]

Di Como CJ, Arndt KT. Nutrients, via the Tor proteins, stimulate the association of Tap42 with type 2A phosphatases. Genes \& development. 1996; 10:1904-1916. [PubMed: 8756348]

Epstein CJ, Smith SA. Amino acid uptake and protein synthesis in preimplanatation mouse embryos. Developmental biology. 1973; 33:171-184. [PubMed: 4789598]

Erbay E, Chen J. The mammalian target of rapamycin regulates $\mathrm{C} 2 \mathrm{C} 12$ myogenesis via a kinaseindependent mechanism. J Biol Chem. 2001; 276:36079-36082. [PubMed: 11500483]

Erbay E, Park IH, Nuzzi PD, Schoenherr CJ, Chen J. IGF-II transcription in skeletal myogenesis is controlled by mTOR and nutrients. J Cell Biol. 2003; 163:931-936. [PubMed: 14662739] 
Fadden P, Haystead TA, Lawrence JC Jr. Identification of phosphorylation sites in the translational regulator, PHAS-I, that are controlled by insulin and rapamycin in rat adipocytes. The Journal of biological chemistry. 1997; 272:10240-10247. [PubMed: 9092573]

Flach G, Johnson MH, Braude PR, Taylor RA, Bolton VN. The transition from maternal to embryonic control in the 2-cell mouse embryo. The EMBO journal. 1982; 1:681-686. [PubMed: 7188357]

Flinn RJ, Yan Y, Goswami S, Parker PJ, Backer JM. The late endosome is essential for mTORC1 signaling. Molecular biology of the cell. 2010; 21:833-841. [PubMed: 20053679]

Fouladi-Nashta AA, Jones CJ, Nijjar N, Mohamet L, Smith A, Chambers I, Kimber SJ. Characterization of the uterine phenotype during the peri-implantation period for LIF-null, MF1 strain mice. Developmental biology. 2005; 281:1-21. [PubMed: 15848385]

Franco HL, Dai D, Lee KY, Rubel CA, Roop D, Boerboom D, Jeong JW, Lydon JP, Bagchi IC, Bagchi MK, DeMayo FJ. ATWNT4 is a key regulator of normal postnatal uterine development and progesterone signaling during embryo implantation and decidualization in the mouse. The FASEB journal : official publication of the Federation of American Societies for Experimental Biology. 2011; 25:1176-1187. [PubMed: 21163860]

Franco HL, Lee KY, Broaddus RR, White LD, Lanske B, Lydon JP, Jeong JW, DeMayo FJ. Ablation of Indian hedgehog in the murine uterus results in decreased cell cycle progression, aberrant epidermal growth factor signaling, and increased estrogen signaling. Biology of reproduction. 2010; 82:783-790. [PubMed: 20056671]

Gangloff YG, Mueller M, Dann SG, Svoboda P, Sticker M, Spetz JF, Um SH, Brown EJ, Cereghini S, Thomas G, Kozma SC. Disruption of the mouse mTOR gene leads to early postimplantation lethality and prohibits embryonic stem cell development. Molecular and cellular biology. 2004; 24:9508-9516. [PubMed: 15485918]

Ge Y, Sun Y, Chen J. IGF-II is regulated by microRNA-125b in skeletal myogenesis. The Journal of cell biology. 2011; 192:69-81. [PubMed: 21200031]

Gingras AC, Gygi SP, Raught B, Polakiewicz RD, Abraham RT, Hoekstra MF, Aebersold R, Sonenberg N. Regulation of 4E-BP1 phosphorylation: a novel two-step mechanism. Genes \& development. 1999; 13:1422-1437. [PubMed: 10364159]

Glantz, S. Primer of Biostatistics. McGraw-Hill, editor. San Francisco, CA: 1992. p. 438

Guertin DA, Stevens DM, Thoreen CC, Burds AA, Kalaany NY, Moffat J, Brown M, Fitzgerald KJ, Sabatini DM. Ablation in mice of the mTORC components raptor, rictor, or mLST8 reveals that mTORC2 is required for signaling to Akt-FOXO and PKCalpha, but not S6K1. Developmental cell. 2006; 11:859-871. [PubMed: 17141160]

Gulhati P, Bowen KA, Liu J, Stevens PD, Rychahou PG, Chen M, Lee EY, Weiss HL, O'Connor KL, Gao T, Evers BM. mTORC1 and mTORC2 regulate EMT, motility, and metastasis of colorectal cancer via RhoA and Rac1 signaling pathways. Cancer research. 2011; 71:3246-3256. [PubMed: 21430067]

Gwatkin RB. Defined media and development of mammalian eggs in vitro. Annals of the New York Academy of Sciences. 1966; 139:79-90. [PubMed: 4290121]

Hamatani T, Daikoku T, Wang H, Matsumoto H, Carter MG, Ko MS, Dey SK. Global gene expression analysis identifies molecular pathways distinguishing blastocyst dormancy and activation. Proceedings of the National Academy of Sciences of the United States of America. 2004; 101:10326-10331. [PubMed: 15232000]

Hara K, Maruki Y, Long X, Yoshino K, Oshiro N, Hidayat S, Tokunaga C, Avruch J, Yonezawa K. Raptor, a binding partner of target of rapamycin (TOR), mediates TOR action. Cell. 2002; 110:177-189. [PubMed: 12150926]

Harris SE, Gopichandran N, Picton HM, Leese HJ, Orsi NM. Nutrient concentrations in murine follicular fluid and the female reproductive tract. Theriogenology. 2005; 64:992-1006. [PubMed: 16054501]

Hernandez-Negrete I, Carretero-Ortega J, Rosenfeldt H, Hernandez-Garcia R, Calderon-Salinas JV, Reyes-Cruz G, Gutkind JS, Vazquez-Prado J. P-Rex1 links mammalian target of rapamycin signaling to Rac activation and cell migration. The Journal of biological chemistry. 2007; 282:23708-23715. [PubMed: 17565979] 
Hillman N, Sherman MI, Graham C. The effect of spatial arrangement on cell determination during mouse development. Journal of embryology and experimental morphology. 1972; 28:263-278. [PubMed: 4674567]

Kaliman P, Canicio J, Testar X, Palacin M, Zorzano A. Insulin-like growth factor-II, phosphatidylinositol 3-kinase, nuclear factor-kappaB and inducible nitric-oxide synthase define a common myogenic signaling pathway. The Journal of biological chemistry. 1999; 274:1743717444. [PubMed: 10364173]

Kanai-Azuma M, Kanai Y, Kurohmaru M, Sakai S, Hayashi Y. Insulin-like growth factor (IGF)-I stimulates proliferation and migration of mouse ectoplacental cone cells, while IGF-II transforms them into trophoblastic giant cells in vitro. Biol Reprod. 1993; 48:252-261. [PubMed: 8439614]

Kaur K, Yao J, Pan X, Matthews C, Hassid A. NO decreases phosphorylation of focal adhesion proteins via reduction of $\mathrm{Ca}$ in rat aortic smooth muscle cells. The American journal of physiology. 1998; 274:H1613-H1619. [PubMed: 9612371]

Kim DH, Sarbassov DD, Ali SM, King JE, Latek RR, Erdjument-Bromage H, Tempst P, Sabatini DM. mTOR interacts with raptor to form a nutrient-sensitive complex that signals to the cell growth machinery. Cell. 2002; 110:163-175. [PubMed: 12150925]

Kim JY, Burghardt RC, Wu G, Johnson GA, Spencer TE, Bazer FW. Select nutrients in the ovine uterine lumen. VII. Effects of arginine, leucine, glutamine, and glucose on trophectoderm cell signaling, proliferation, and migration. Biology of reproduction. 2011; 84:62-69. [PubMed: 20844282]

Koromilas AE, Lazaris-Karatzas A, Sonenberg N. mRNAs containing extensive secondary structure in their 5 ' non-coding region translate efficiently in cells overexpressing initiation factor eIF-4E. The EMBO journal. 1992; 11:4153-4158. [PubMed: 1396596]

Kunz J, Henriquez R, Schneider U, Deuter-Reinhard M, Movva NR, Hall MN. Target of rapamycin in yeast, TOR2, is an essential phosphatidylinositol kinase homolog required for G1 progression. Cell. 1993; 73:585-596. [PubMed: 8387896]

Laplante M, Sabatini DM. An emerging role of mTOR in lipid biosynthesis. Current biology : CB. 2009a; 19:R1046-R1052. [PubMed: 19948145]

Laplante M, Sabatini DM. mTOR signaling at a glance. Journal of cell science. 2009b; 122:35893594. [PubMed: 19812304]

Large MJ, Demayo FJ. The regulation of embryo implantation and endometrial decidualization by progesterone receptor signaling. Mol Cell Endocrinol. 2011

Lazaris-Karatzas A, Montine KS, Sonenberg N. Malignant transformation by a eukaryotic initiation factor subunit that binds to mRNA 5' cap. Nature. 1990; 345:544-547. [PubMed: 2348862]

Lee JE, Pintar J, Efstratiadis A. Pattern of the insulin-like growth factor II gene expression during early mouse embryogenesis. Development (Cambridge, England). 1990; 110:151-159.

Lee K, Jeong J, Kwak I, Yu CT, Lanske B, Soegiarto DW, Toftgard R, Tsai MJ, Tsai S, Lydon JP, DeMayo FJ. Indian hedgehog is a major mediator of progesterone signaling in the mouse uterus. Nature genetics. 2006; 38:1204-1209. [PubMed: 16951680]

Levey IL, Brinster RL. Effects of alpha-amanitin on RNA synthesis by mouse embryos in culture. The Journal of experimental zoology. 1978; 203:351-360. [PubMed: 641476]

Levine JE, Pau KY, Ramirez VD, Jackson GL. Simultaneous measurement of luteinizing hormonereleasing hormone and luteinizing hormone release in unanesthetized, ovariectomized sheep. Endocrinology. 1982; 111:1449-1455. [PubMed: 6751794]

Levine JE, Ramirez VD. Luteinizing hormone-releasing hormone release during the rat estrous cycle and after ovariectomy, as estimated with push-pull cannulae. Endocrinology. 1982; 111:14391448. [PubMed: 6751793]

Lin TA, Kong X, Haystead TA, Pause A, Belsham G, Sonenberg N, Lawrence JC Jr. PHAS-I as a link between mitogen-activated protein kinase and translation initiation. Science (New York, N.Y.). 1994; 266:653-656.

Lindell TJ, Weinberg F, Morris PW, Roeder RG, Rutter WJ. Specific inhibition of nuclear RNA polymerase II by alpha-amanitin. Science (New York, N.Y.). 1970; 170:447-449. 
Liu L, Li F, Cardelli JA, Martin KA, Blenis J, Huang S. Rapamycin inhibits cell motility by suppression of mTOR-mediated S6K1 and 4E-BP1 pathways. Oncogene. 2006; 25:7029-7040. [PubMed: 16715128]

Lubahn DB, Moyer JS, Golding TS, Couse JF, Korach KS, Smithies O. Alteration of reproductive function but not prenatal sexual development after insertional disruption of the mouse estrogen receptor gene. Proceedings of the National Academy of Sciences of the United States of America. 1993; 90:11162-11166. [PubMed: 8248223]

Lydon JP, DeMayo FJ, Funk CR, Mani SK, Hughes AR, Montgomery CA Jr, Shyamala G, Conneely OM, O'Malley BW. Mice lacking progesterone receptor exhibit pleiotropic reproductive abnormalities. Genes \& development. 1995; 9:2266-2278. [PubMed: 7557380]

Martin PM, Sutherland AE. Exogenous amino acids regulate trophectoderm differentiation in the mouse blastocyst through an mTOR-dependent pathway. Developmental biology. 2001; 240:182193. [PubMed: 11784055]

Martin PM, Sutherland AE, Van Winkle LJ. Amino acid transport regulates blastocyst implantation. Biology of reproduction. 2003; 69:1101-1108. [PubMed: 12801981]

Midgley AR Jr, Jaffe RB. Regulation of human gonadotropins. X. Episodic fluctuation of LH during the menstrual cycle. The Journal of clinical endocrinology and metabolism. 1971; 33:962-969. [PubMed: 5135635]

Mothe-Satney I, Brunn GJ, McMahon LP, Capaldo CT, Abraham RT, Lawrence JC Jr. Mammalian target of rapamycin-dependent phosphorylation of PHAS-I in four (S/T)P sites detected by phospho-specific antibodies. J Biol Chem. 2000a; 275:33836-33843. [PubMed: 10942774]

Mothe-Satney I, Yang D, Fadden P, Haystead TA, Lawrence JC Jr. Multiple mechanisms control phosphorylation of PHAS-I in five (S/T)P sites that govern translational repression. Mol Cell Biol. 2000b; 20:3558-3567. [PubMed: 10779345]

Naeslund G. The effect of glucose-, arginine- and leucine-deprivation on mouse blastocyst outgrowth in vitro. Upsala journal of medical sciences. 1979; 84:9-20. [PubMed: 442281]

Nicklin P, Bergman P, Zhang B, Triantafellow E, Wang H, Nyfeler B, Yang H, Hild M, Kung C, Wilson C, Myer VE, MacKeigan JP, Porter JA, Wang YK, Cantley LC, Finan PM, Murphy LO. Bidirectional transport of amino acids regulates mTOR and autophagy. Cell. 2009; 136:521-534. [PubMed: 19203585]

Nilsson BO, Ljung L. X-ray micro analyses of cations (Na, K, Ca) and anions (, , P, Cl) in uterine secretions during blastocyst implantation in the rat. The Journal of experimental zoology. 1985; 234:415-421. [PubMed: 4056679]

Palacin M, Nunes V, Font-Llitjos M, Jimenez-Vidal M, Fort J, Gasol E, Pineda M, Feliubadalo L, Chillaron J, Zorzano A. The genetics of heteromeric amino acid transporters. Physiology (Bethesda, Md). 2005; 20:112-124.

Paria BC, Das SK, Andrews GK, Dey SK. Expression of the epidermal growth factor receptor gene is regulated in mouse blastocysts during delayed implantation. Proceedings of the National Academy of Sciences of the United States of America. 1993a; 90:55-59. [PubMed: 7678348]

Paria BC, Huet-Hudson YM, Dey SK. Blastocyst's state of activity determines the "window" of implantation in the receptive mouse uterus. Proceedings of the National Academy of Sciences of the United States of America. 1993b; 90:10159-10162. [PubMed: 8234270]

Pause A, Belsham GJ, Gingras AC, Donze O, Lin TA, Lawrence JC Jr, Sonenberg N. Insulindependent stimulation of protein synthesis by phosphorylation of a regulator of 5'-cap function. Nature. 1994; 371:762-767. [PubMed: 7935836]

Pendeville H, Carpino N, Marine JC, Takahashi Y, Muller M, Martial JA, Cleveland JL. The ornithine decarboxylase gene is essential for cell survival during early murine development. Molecular and cellular biology. 2001; 21:6549-6558. [PubMed: 11533243]

Psychoyos, A. Endocrine control of egg implantation. In: GROaG, SR., editor. Handbook of Physiology. Washington, D.C.: American Physiological Society; 1973a. p. 187-215.

Psychoyos A. Hormonal control of ovoimplantation. Vitamins and hormones. 1973b; 31:201-256. [PubMed: 4620375] 
Raab G, Kover K, Paria BC, Dey SK, Ezzell RM, Klagsbrun M. Mouse preimplantation blastocysts adhere to cells expressing the transmembrane form of heparin-binding EGF-like growth factor. Development (Cambridge, England). 1996; 122:637-645.

Ramathal CY, Bagchi IC, Taylor RN, Bagchi MK. Endometrial decidualization: of mice and men. Seminars in reproductive medicine. 2010; 28:17-26. [PubMed: 20104425]

Sancak Y, Bar-Peled L, Zoncu R, Markhard AL, Nada S, Sabatini DM. Ragulator-Rag complex targets mTORC1 to the lysosomal surface and is necessary for its activation by amino acids. Cell. 2010; 141:290-303. [PubMed: 20381137]

Sancak Y, Peterson TR, Shaul YD, Lindquist RA, Thoreen CC, Bar-Peled L, Sabatini DM. The Rag GTPases bind raptor and mediate amino acid signaling to mTORC1. Science (New York, N.Y.). 2008; 320:1496-1501.

Santen RJ, Bardin CW. Episodic luteinizing hormone secretion in man. Pulse analysis, clinical interpretation, physiologic mechanisms. The Journal of clinical investigation. 1973; 52:26172628. [PubMed: 4729055]

Sarbassov DD, Ali SM, Kim DH, Guertin DA, Latek RR, Erdjument-Bromage H, Tempst P, Sabatini DM. Rictor, a novel binding partner of $\mathrm{mTOR}$, defines a rapamycin-insensitive and raptorindependent pathway that regulates the cytoskeleton. Current biology : CB. 2004; 14:1296-1302. [PubMed: 15268862]

Schultz JF, Armant DR. Beta 1- and beta 3-class integrins mediate fibronectin binding activity at the surface of developing mouse peri-implantation blastocysts. Regulation by ligand-induced mobilization of stored receptor. The Journal of biological chemistry. 1995; 270:11522-11531. [PubMed: 7538116]

Schultz JF, Mayernik L, Rout UK, Armant DR. Integrin trafficking regulates adhesion to fibronectin during differentiation of mouse peri-implantation blastocysts. Developmental genetics. 1997; 21:31-43. [PubMed: 9291578]

Sengoku K, Takuma N, Horikawa M, Tsuchiya K, Komori H, Sharifa D, Tamate K, Ishikawa M. Requirement of nitric oxide for murine oocyte maturation, embryo development, and trophoblast outgrowth in vitro. Molecular reproduction and development. 2001; 58:262-268. [PubMed: 11170266]

Shama S, Meyuhas O. The translational cis-regulatory element of mammalian ribosomal protein mRNAs is recognized by the plant translational apparatus. European journal of biochemistry/ FEBS. 1996; 236:383-388. [PubMed: 8612606]

Shen MM, Leder P. Leukemia inhibitory factor is expressed by the preimplantation uterus and selectively blocks primitive ectoderm formation in vitro. Proceedings of the National Academy of Sciences of the United States of America. 1992; 89:8240-8244. [PubMed: 1518852]

Sloan JL. Cloning and charaterization of the amino acid transporter $\operatorname{ATB}(0,+)$, Neurobiology. University of North Carolina at Chapel Hill, Chapel Hill. 2002:193.

Sloan JL, Mager S. Cloning and functional expression of a human $\mathrm{Na}(+)$ and $\mathrm{Cl}(-)$-dependent neutral and cationic amino acid transporter B(0+). The Journal of biological chemistry. 1999; 274:23740 23745. [PubMed: 10446133]

Song H, Lim H, Das SK, Paria BC, Dey SK. Dysregulation of EGF family of growth factors and COX-2 in the uterus during the preattachment and attachment reactions of the blastocyst with the luminal epithelium correlates with implantation failure in LIF-deficient mice. Molecular endocrinology (Baltimore, Md). 2000; 14:1147-1161.

Song H, Lim H, Paria BC, Matsumoto H, Swift LL, Morrow J, Bonventre JV, Dey SK. Cytosolic phospholipase A2alpha is crucial [correction of A2alpha deficiency is crucial] for 'on-time' embryo implantation that directs subsequent development. Development (Cambridge, England). 2002; 129:2879-2889.

Spindle A. An improved culture medium for mouse blastocysts. In vitro. 1980; 16:669-674. [PubMed: 7419236]

Spindle AI, Pedersen RA. Hatching, attachment, and outgrowth of mouse blastocysts in vitro: fixed nitrogen requirements. The Journal of experimental zoology. 1973; 186:305-318. [PubMed: 4765353] 
Squarize CH, Castilho RM, Bugge TH, Gutkind JS. Accelerated wound healing by mTOR activation in genetically defined mouse models. PloS one. 2010; 5:e10643. [PubMed: 20498714]

Stephens LE, Sutherland AE, Klimanskaya IV, Andrieux A, Meneses J, Pedersen RA, Damsky CH. Deletion of beta 1 integrins in mice results in inner cell mass failure and peri-implantation lethality. Genes \& development. 1995; 9:1883-1895. [PubMed: 7544312]

Stewart CL, Kaspar P, Brunet LJ, Bhatt H, Gadi I, Kontgen F, Abbondanzo SJ. Blastocyst implantation depends on maternal expression of leukaemia inhibitory factor. Nature. 1992; 359:76-79. [PubMed: 1522892]

Sutherland AE, Calarco PG, Damsky CH. Developmental regulation of integrin expression at the time of implantation in the mouse embryo. Development (Cambridge, England). 1993; 119:1175-1186.

Tarkowski AK, Wroblewska J. Development of blastomeres of mouse eggs isolated at the 4- and 8-cell stage. Journal of embryology and experimental morphology. 1967; 18:155-180. [PubMed: 6048976]

Tranguch S, Daikoku T, Guo Y, Wang H, Dey SK. Molecular complexity in establishing uterine receptivity and implantation. Cellular and molecular life sciences : CMLS. 2005; 62:1964-1973. [PubMed: 16143898]

Van Winkle DM, Matsuki T, Gad NM, Jordan MC, Downey JM. Left ventricular unloading during reperfusion does not limit myocardial infarct size. Circulation. 1990a; 81:1374-1379. [PubMed: 2317914]

Van Winkle LJ. Activation of amino acid accumulation in delayed implantation mouse blastocysts. The Journal of experimental zoology. 1981; 218:239-246. [PubMed: 7320688]

Van Winkle LJ. Amino acid transport regulation and early embryo development. Biology of reproduction. 2001; 64:1-12. [PubMed: 11133652]

Van Winkle LJ, Campione AL, Gorman JM. Inhibition of transport system b0, + in blastocysts by inorganic and organic cations yields insight into the structure of its amino acid receptor site. Biochimica et biophysica acta. 1990b; 1025:215-224. [PubMed: 2114171]

Van Winkle LJ, Campione AL, Webster DP. Sodium ion concentrations in uterine flushings from "implanting" and "delayed implanting" mice. The Journal of experimental zoology. 1983; 226:321-324. [PubMed: 6864183]

Van Winkle LJ, Christensen HN, Campione AL. Na+-dependent transport of basic, zwitterionic, and bicyclic amino acids by a broad-scope system in mouse blastocysts. The Journal of biological chemistry. 1985; 260:12118-12123. [PubMed: 3930488]

Van Winkle LJ, Haghighat N, Campione AL, Gorman JM. Glycine transport in mouse eggs and preimplantation conceptuses. Biochimica et biophysica acta. 1988; 941:241-256. [PubMed: 2454661]

Van Winkle LJ, Tesch JK, Shah A, Campione AL. System B0,+ amino acid transport regulates the penetration stage of blastocyst implantation with possible long-term developmental consequences through adulthood. Human reproduction update. 2006; 12:145-157. [PubMed: 16251251]

Wakitani S, Hondo E, Phichitraslip T, Stewart CL, Kiso Y. Upregulation of Indian hedgehog gene in the uterine epithelium by leukemia inhibitory factor during mouse implantation. The Journal of reproduction and development. 2008; 54:113-116. [PubMed: 18239353]

Wang H, Ma WG, Tejada L, Zhang H, Morrow JD, Das SK, Dey SK. Rescue of female infertility from the loss of cyclooxygenase- 2 by compensatory up-regulation of cyclooxygenase- 1 is a function of genetic makeup. The Journal of biological chemistry. 2004; 279:10649-10658. [PubMed: 14701858]

Wang H, Xie H, Sun X, Tranguch S, Zhang H, Jia X, Wang D, Das SK, Desvergne B, Wahli W, DuBois RN, Dey SK. Stage-specific integration of maternal and embryonic peroxisome proliferator-activated receptor delta signaling is critical to pregnancy success. The Journal of biological chemistry. 2007; 282:37770-37782. [PubMed: 17965409]

Wang J, Mayernik L, Armant DR. Integrin signaling regulates blastocyst adhesion to fibronectin at implantation: intracellular calcium transients and vesicle trafficking in primary trophoblast cells. Developmental biology. 2002; 245:270-279. [PubMed: 11977980]

Wang J, Mayernik L, Schultz JF, Armant DR. Acceleration of trophoblast differentiation by heparinbinding EGF-like growth factor is dependent on the stage-specific activation of calcium influx by 
ErbB receptors in developing mouse blastocysts. Development (Cambridge, England). 2000; 127:33-44.

Wang XN, Das SK, Damm D, Klagsbrun M, Abraham JA, Dey SK. Differential regulation of heparinbinding epidermal growth factor-like growth factor in the adult ovariectomized mouse uterus by progesterone and estrogen. Endocrinology. 1994; 135:1264-1271. [PubMed: 8070372]

Wilcox AJ, Baird DD, Weinberg CR. Time of implantation of the conceptus and loss of pregnancy. The New England journal of medicine. 1999; 340:1796-1799. [PubMed: 10362823]

Xie H, Tranguch S, Jia X, Zhang H, Das SK, Dey SK, Kuo CJ, Wang H. Inactivation of nuclear Wntbeta-catenin signaling limits blastocyst competency for implantation. Development (Cambridge, England). 2008; 135:717-727.

Yang Q, Guan KL. Expanding mTOR signaling. Cell Res. 2007; 17:666-681. [PubMed: 17680028]

Ye X, Hama K, Contos JJ, Anliker B, Inoue A, Skinner MK, Suzuki H, Amano T, Kennedy G, Arai H, Aoki J, Chun J. LPA3-mediated lysophosphatidic acid signalling in embryo implantation and spacing. Nature. 2005; 435:104-108. [PubMed: 15875025]

Yelian FD, Yang Y, Hirata JD, Schultz JF, Armant DR. Molecular interactions between fibronectin and integrins during mouse blastocyst outgrowth. Molecular reproduction and development. 1995; 41:435-448. [PubMed: 7576611]

Yoshinaga K, Adams CE. Delayed implantation in the spayed, progesterone treated adult mouse. Journal of reproduction and fertility. 1966; 12:593-595. [PubMed: 5928277]

Yoshinaga K, Hawkins RA, Stocker JF. Estrogen secretion by the rat ovary in vivo during the estrous cycle and pregnancy. Endocrinology. 1969; 85:103-112. [PubMed: 5815010]

Zhao YC, Chi YJ, Yu YS, Liu JL, Su RW, Ma XH, Shan CH, Yang ZM. Polyamines are essential in embryo implantation: expression and function of polyamine-related genes in mouse uterus during peri-implantation period. Endocrinology. 2008; 149:2325-2332. [PubMed: 18202119]

Ziomek CA, Johnson MH. Cell surface interaction induces polarization of mouse 8-cell blastomeres at compaction. Cell. 1980; 21:935-942. [PubMed: 7438209] 

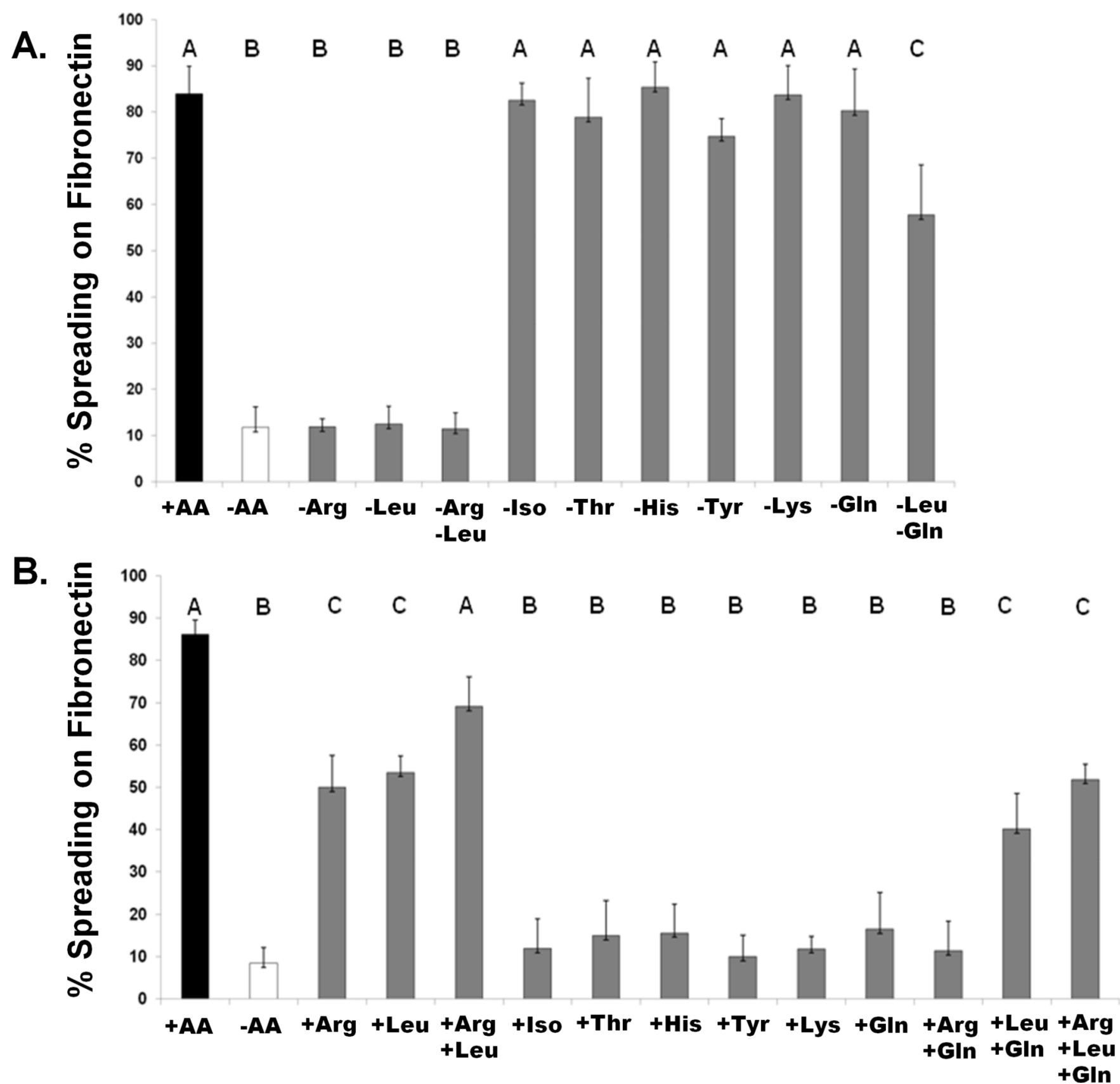

Figure 1. Arginine and leucine are necessary and sufficient for trophoblast outgrowth

A. Graph showing the percent of embryos exhibiting any trophoblast outgrowth following 8 hours of culture in medium containing all amino acids except the indicated ones: arginine and/or leucine (-Arg, -Leu, -Arg-Leu); Isoleucine (-Iso), Threonine (-Thr), Histidine (-His), Tyrosine, (-Tyr), Lysine (-Lys), Glutamine (-Gln).

B. Graph showing the percent of embryos exhibiting any trophoblast outgrowth following 8 hours of culture in medium containing no amino acids except the indicated ones: $0.2 \mathrm{mM}$ arginine $(+\mathrm{Arg})$ or $0.2 \mathrm{mM}$ leucine $(+\mathrm{Leu})$ or both in 
combination (+Arg+Leu); Isoleucine (+Iso), Threonine (+Thr), Histidine (+His), Tyrosine, (+Tyr), Lysine (+Lys), Glutamine (+Gln).

For both graphs A and B, the data represent the mean +/- SEM for 6 independent experiments. Mean values labeled with different letters are significantly different from one another, $\mathrm{P} \leq 0.01$ 
A.

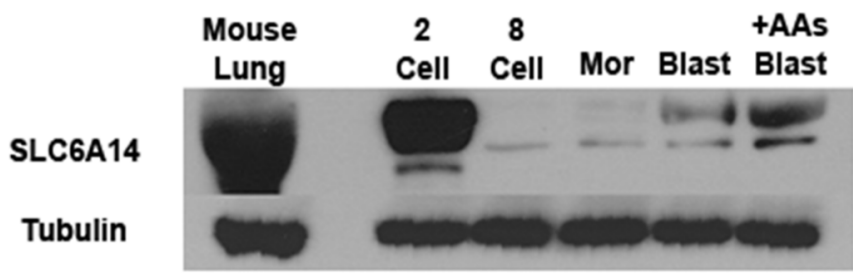

C.

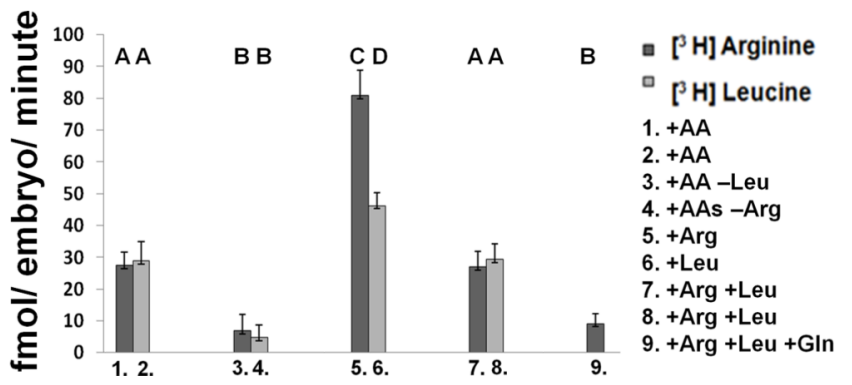

B.
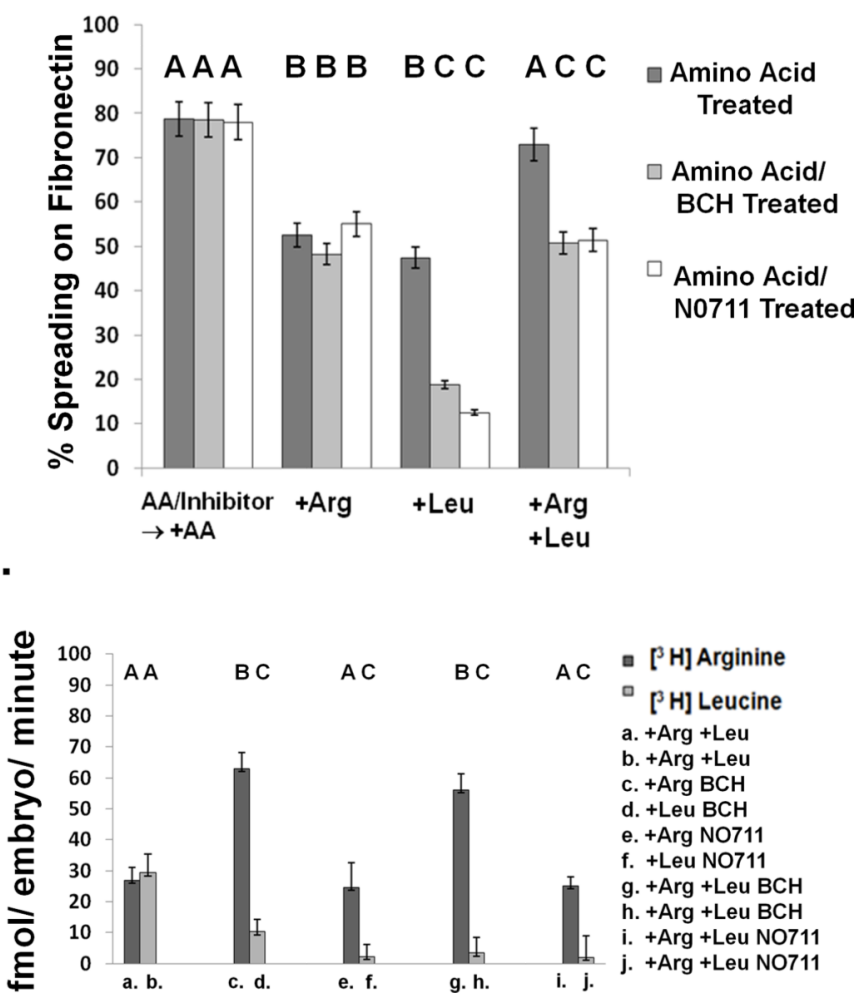

Figure 2. SLC6A14 protein expression is temporally regulated and its activity is critical for trophoblast outgrowth

A. Western blot of whole cell lysates of 100 embryos at the indicated stages, and of normal mouse lung as a control, probed with an antibody against SLC6A14 then probed with an antibody to tubulin as a loading control. (Mor: Morula, Blast:Blastocyst)

B. Graph showing the percent of embryos exhibiting any trophoblast outgrowth following 8 hours incubation in the media indicated. The data in the first three bars represent toxicity controls, where embryos were incubated for 8 hours in $+\mathrm{AA}$ medium with or without $\mathrm{NO} 711$ or $\mathrm{BCH}$, washed and then transferred to +AA medium on fibronectin. All other embryos were incubated for 8 hours in medium containing the indicated amino acids, with or without $\mathrm{NO} 711$ or $\mathrm{BCH}$, and then transferred into - AA medium on fibronectin. The data represent the mean $+/-$ SEM of 6 independent experiments. Mean values labeled with different letters are significantly different from one another, $(\mathrm{p} \leq 0.01)$.

C. Blastocyst uptake of $\left[{ }^{3} \mathrm{H}\right]$ arginine and $\left[{ }^{3} \mathrm{H}\right]$ leucine following 8 hours incubation in the indicated media. The number under each bar corresponds to the number on the list to the right of the graph showing the medium used and the color of the bar represents the amino acid measured in that condition. The data represent the mean $+/-$ SEM of 3 independent experiments performed in triplicate. Mean values labeled with different letters are significantly different from one another, $(\mathrm{p} \leq$ $0.01)$.

D. Blastocyst up take of $\left[{ }^{3} \mathrm{H}\right]$ arginine and $\left[{ }^{3} \mathrm{H}\right]$ leucine following 8 hours incubation in media containing the SLC6A14 inhibitors BCH or NO711. The letter under each bar corresponds to the letter on the list to the right of the graph indicating the 
medium used and the color of the bar represents the amino acid measured in that condition. The data represent the mean +/- SEM of 3 independent experiments performed in triplicate. Mean values labeled with different letters are significantly different from one another, $(\mathrm{p} \leq 0.01)$. 
A.

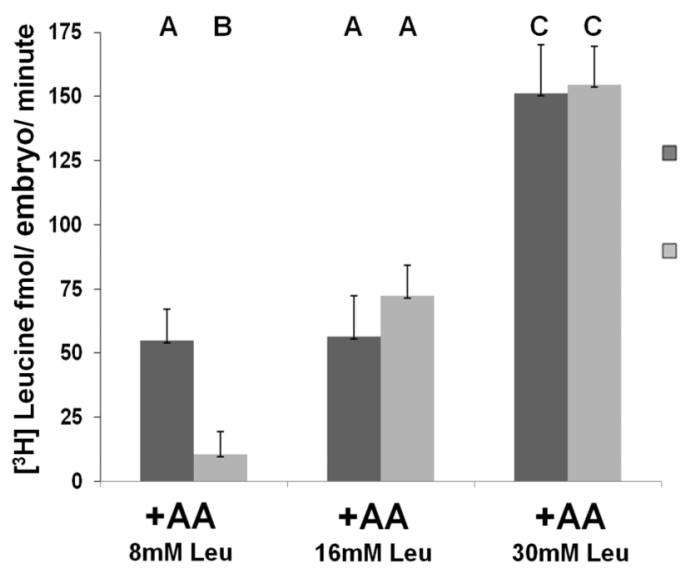

B.

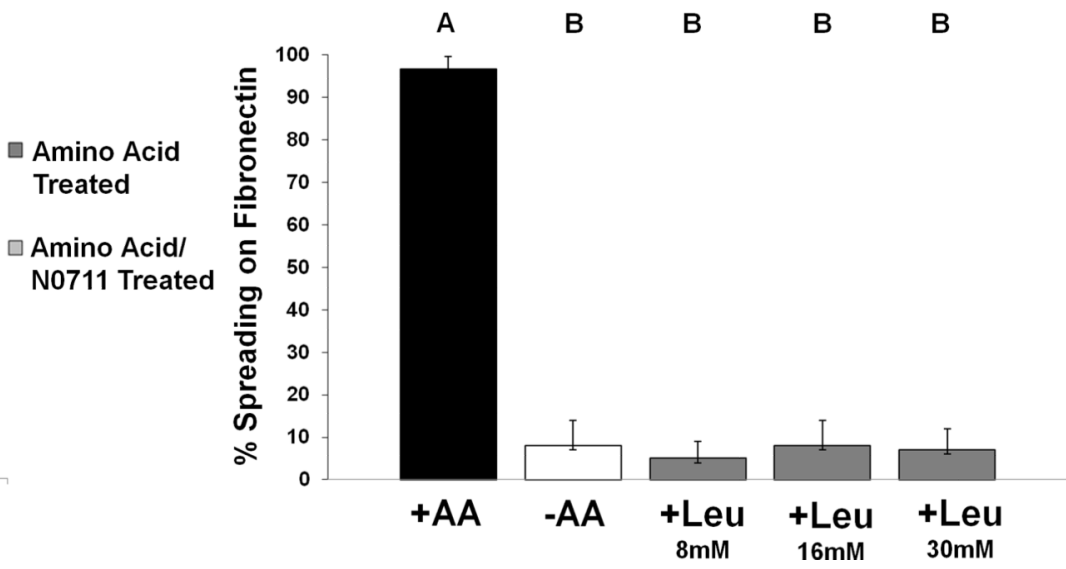

Figure 3. Increased leucine uptake via other amino acid transporters does not produce trophoblast outgrowth

A. Graph showing mean uptake of $\left[{ }^{3} \mathrm{H}\right]$ leucine by blastocysts following 8 hours incubation in medium containing increasing concentrations of leucine with or without NO711.

The data represent the mean $+/-$ SEM of 3 independent experiments performed in triplicate.

Bars labeled with different letters are significantly different from one another, $(\mathrm{p} \leq$ $0.01)$

B. Graph showing percent of embryos exhibiting any trophoblast outgrowth after 8 hours of incubation in the indicated medium.

For both graphs, the data represent the mean $+/-$ SEM of 3 independent experiments performed in triplicate.

Mean values labeled with different letters are significantly different from one another, $(\mathrm{p} \leq$ $0.01)$ 

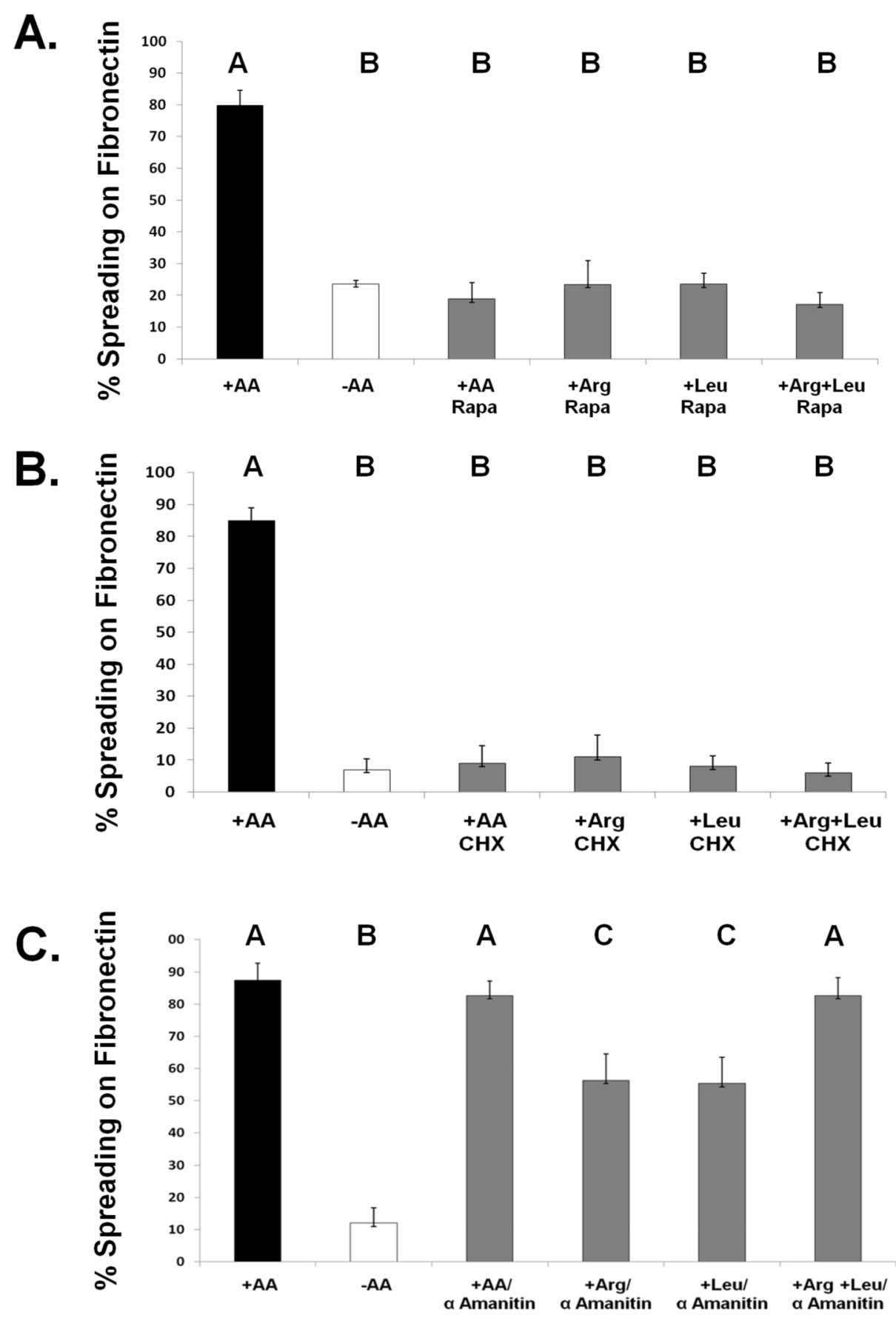

Figure 4. mTOR activation and protein translation are necessary for arginine- or leucinestimulated trophoblast outgrowth, but RNA transcription is not

A. Graph showing the percent of embryos exhibiting trophoblast outgrowth following 8 hours incubation in medium containing the indicated amino acids in combination with rapamycin (Rapa).

B. Graph showing the percent of embryos exhibiting trophoblast outgrowth following 8 hours incubation in medium containing the indicated amino acids in combination with cycloheximide (CHX). 
C. Graph showing the percent of embryos exhibiting trophoblast outgrowth following 8 hours incubation in medium containing the indicated amino acids in combination with alpha-amanitin.

For all graphs, the data represent the mean $+/-$ SEM from 4 independent experiments. Mean values labeled with different letters are significantly different from one another $(p \leq 0.01)$. 
A.

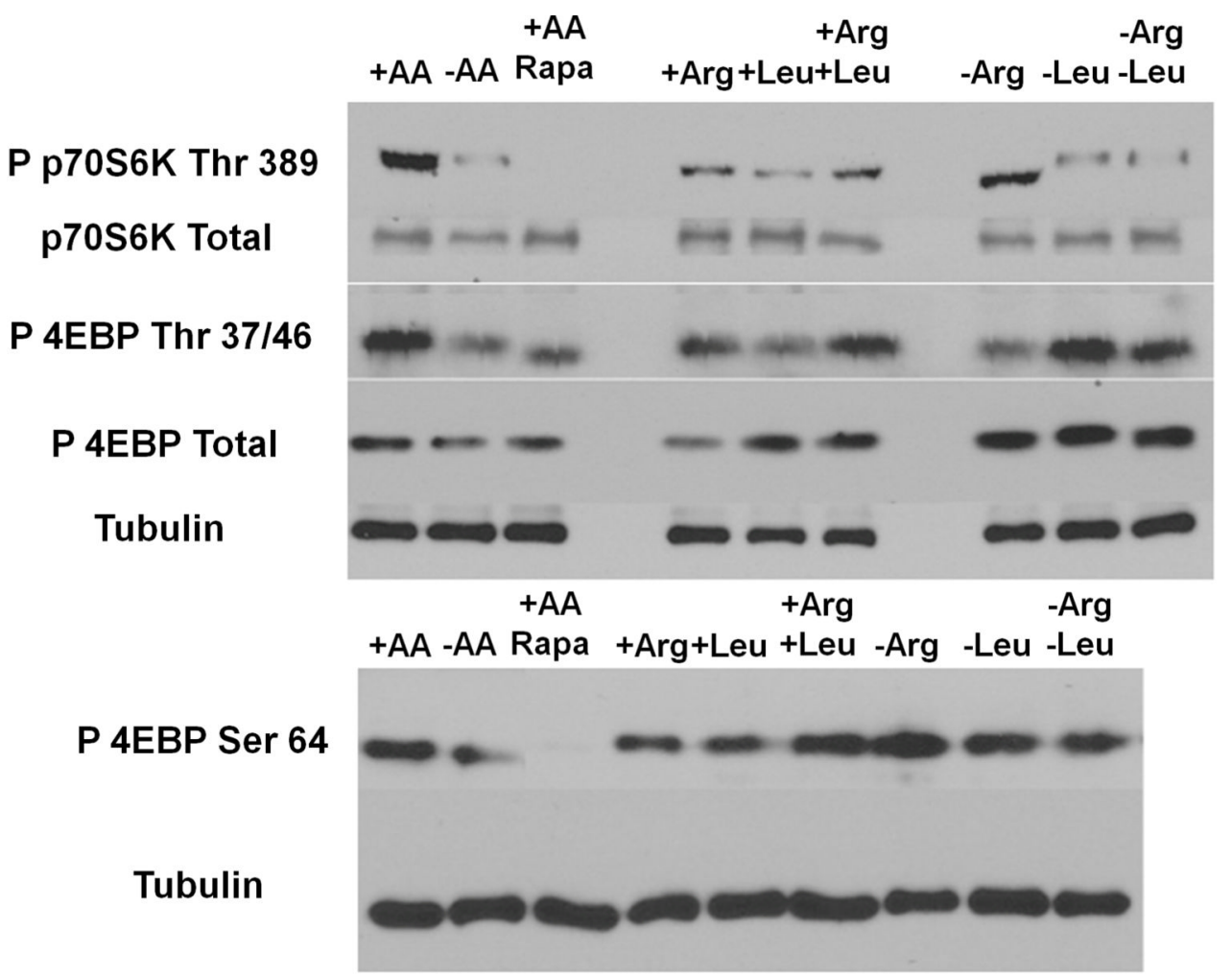

C.

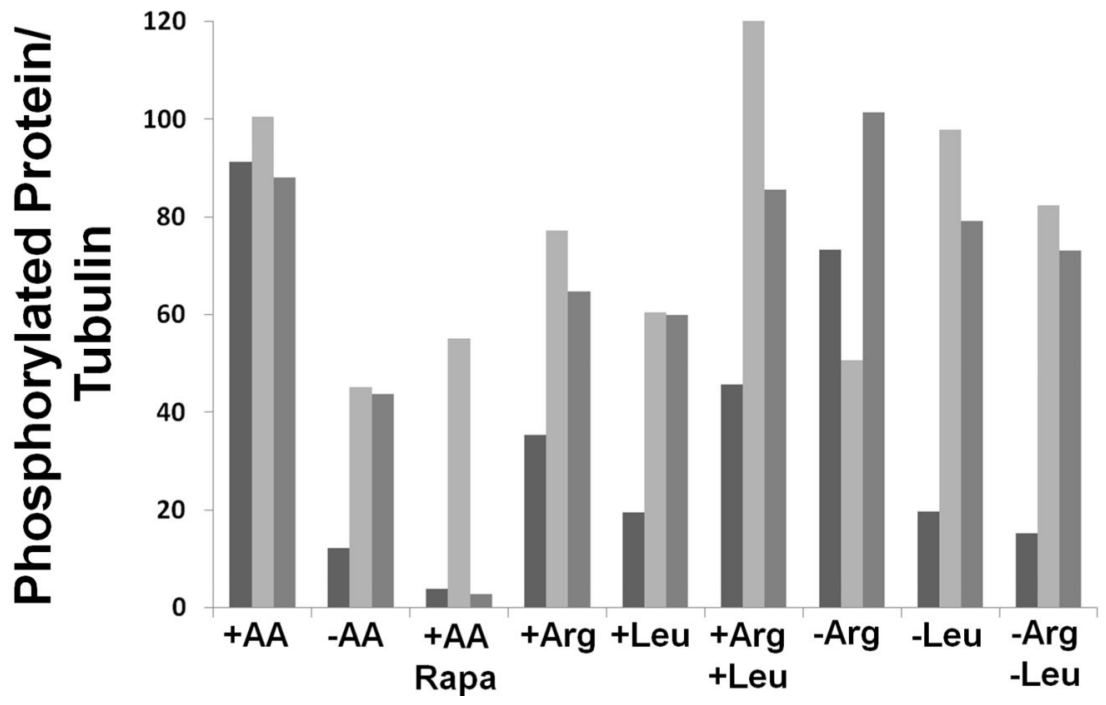

- p70S6K Thr 389

4EBP Thr $37 / 46$

4EBP Thr 64

Figure 5. Phosphorylation of TORC1 downstream effectors p70S6K or 4EBP does not correlate with embryo spreading

A. Western blot of whole-cell lysates of 100 embryos (per lane) cultured for 8 hours in the indicated medium, probed sequentially for phospho-Thr389 p70S6K and phospho-Thr37/46 4EBP, then for the total amount of p70S6K and 4EBP, and finally for tubulin as a loading control.

B. Western blot of whole-cell lysates of 100 embryos cultured for 8 hours in the indicated medium, probed sequentially for phospho-Ser64 4EBP and for tubulin as a loading control. 
C. Graphical representation of the percentage of p70S6K that is phosphorylated at Thr 389 and of 4EBP that is phosphorylated at Thr37/46 or Ser 64, normalized to tubulin for each sample. These values are derived from quantitation of the blots shown in $\mathrm{A}$ and $\mathrm{B}$, which are consistent with other blots done on these same treatment groups. 


\section{8 \\ OocyteCell Cell Mor Blast}

mTOR Rictor Raptor

LST8

\section{Tubulin}

Rcho-1

WCL

293s

WCL

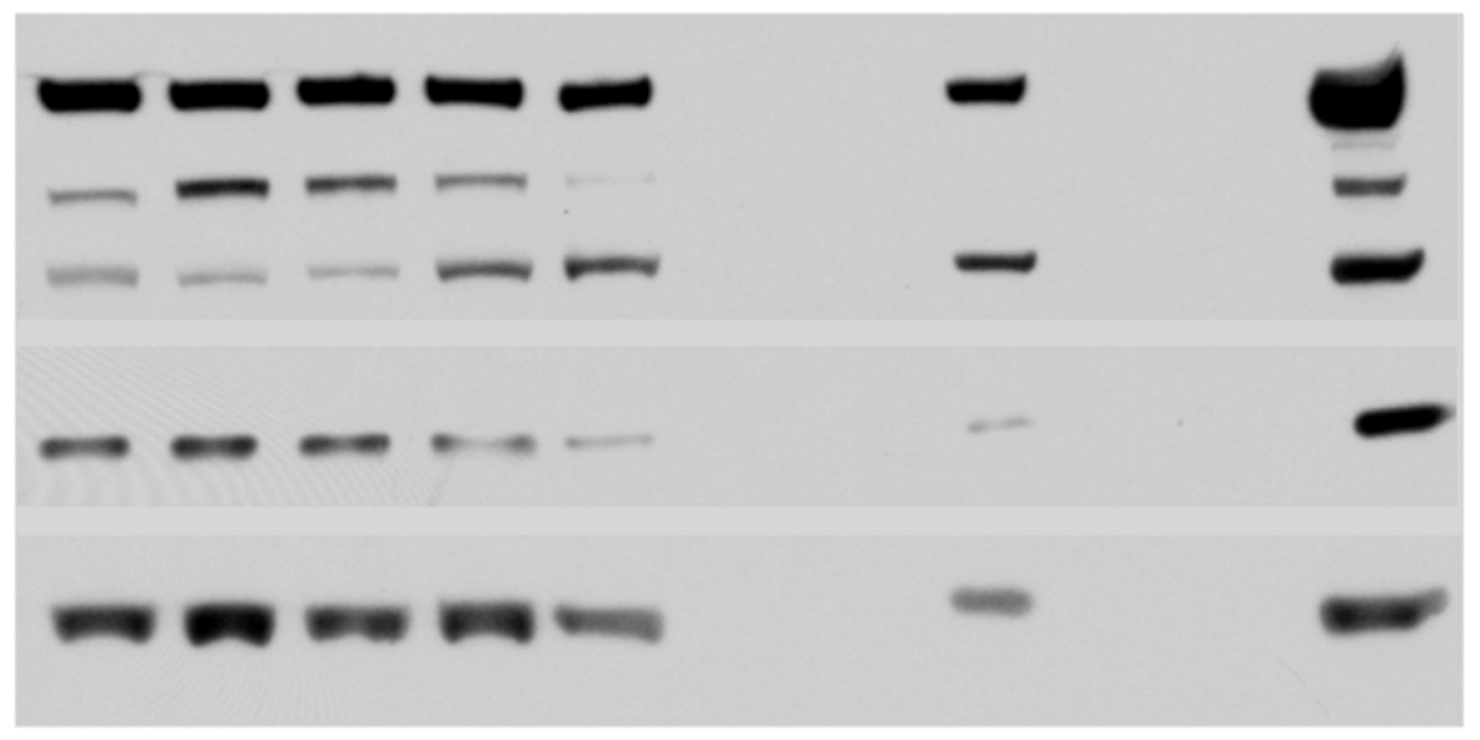

Figure 6. The TORC1 components mTOR and Raptor are preferentially expressed in the blastocyst

Western blot of whole-cell lysates (WCL) of 100 embryos (per lane) at the indicated stages, and of the rat choriocarcinoma cell line (Rcho-1) and the human embryonic kidney cell line S293 (293S), probed simultaneously with antibodies against mTOR, Rictor, Raptor, and LST8, and then probed with an antibody against tubulin as a loading control. (Mor, Morula; Blast, Blastocysts) 

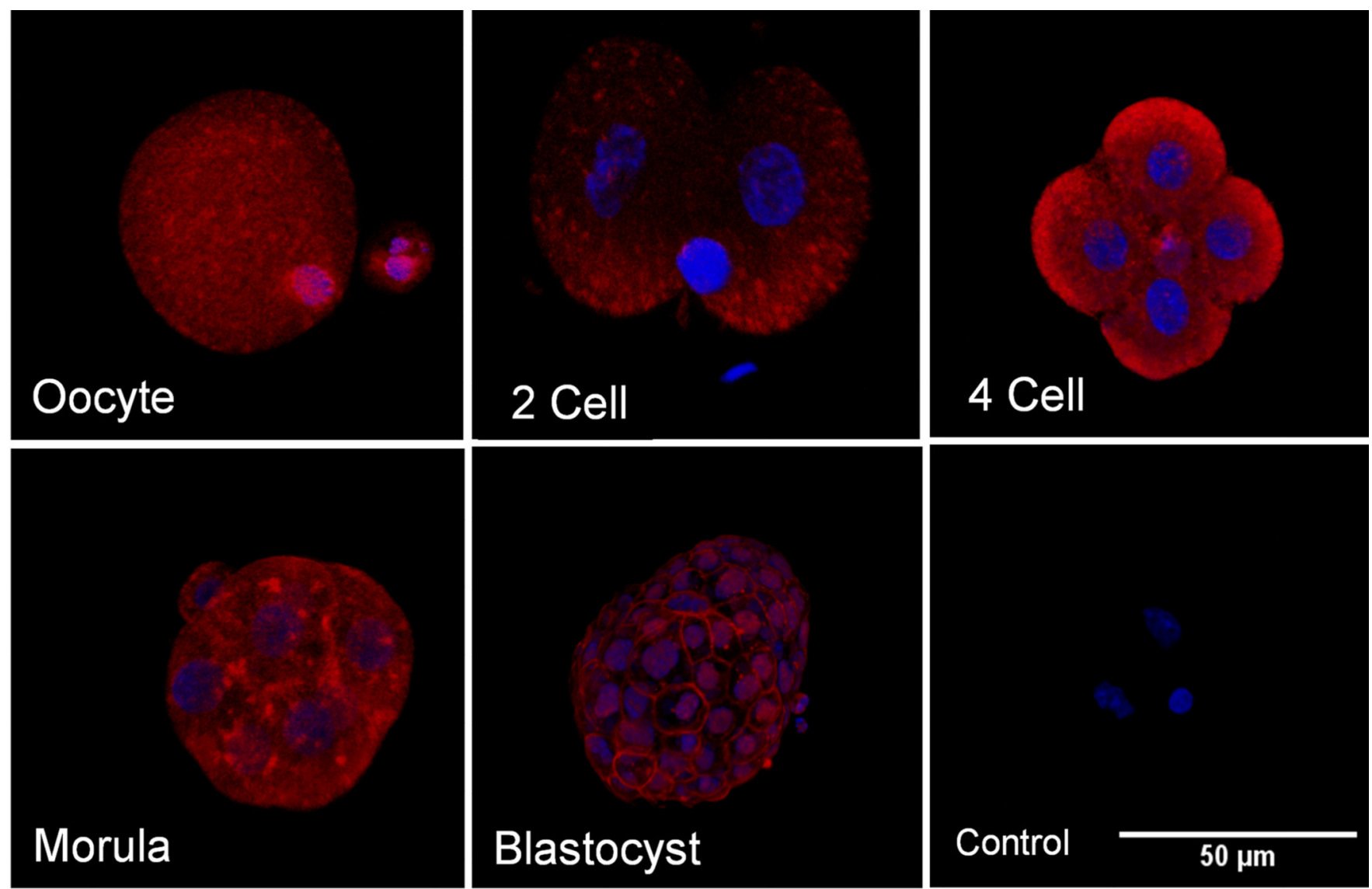

Figure 7. mTOR localization in oocytes and peri-implantation embryos

Immunofluorescent localization of mTOR and DAPI within the oocyte, and in embryos at 48h (2-cell) and 68h (8-cell), 96h (morula), and 120h phCG (blastocyst). The images are extended focus composites of confocal Z-stacks. Control is secondary antibody alone with DAPI staining. 

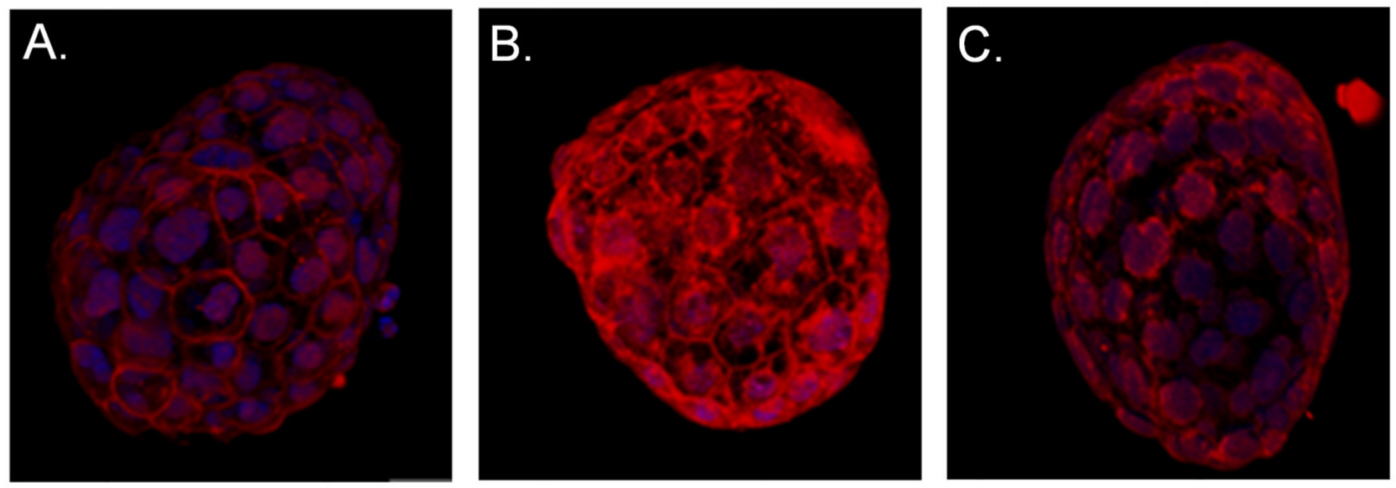

J.
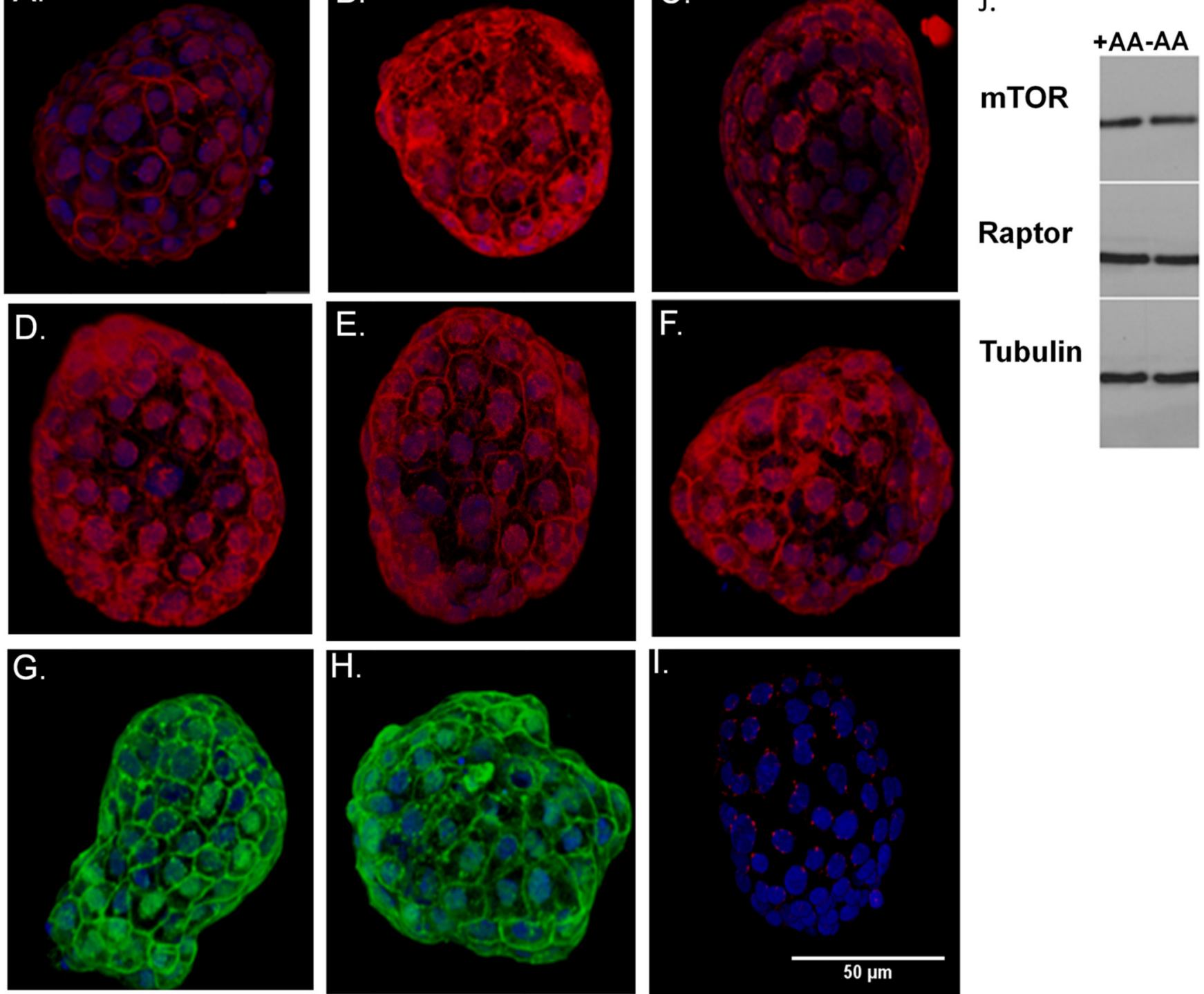

Figure 8. Immunofluorescent signal intensity from Cell Signaling Technology (CST) antibody \#2983 increases upon amino acid-stimulated mTOR activation
A. Unstimulated (-AA, 8 hours) $120 \mathrm{~h}$ phCG blastocyst...
B. Amino acid stimulated (+AA, 8 hours) $120 \mathrm{~h}$ phCG blastocyst.
C. Amino acid and Rapamycin (+AA/Rapamycin, 8 hours) treated $120 \mathrm{~h}$ phCG blastocyst.
D. Increased mTOR signal intensity in $120 \mathrm{~h}$ phCG embryo stimulated with amino acids for 1'.
E. Increased mTOR signal intensity in $120 \mathrm{~h}$ phCG embryo stimulated with amino acids for 5 '.
F. Increased mTOR signal intensity in $120 \mathrm{~h}$ phCG embryo stimulated with amino acids for 10 '.


G. Unstimulated ( $-\mathrm{AA}, 8$ hours) $120 \mathrm{~h}$ phCG blastocyst stained with antibody to Raptor.

H. Amino acid stimulated (+AA, 8 hours) $120 \mathrm{~h}$ phCG blastocyst stained with antibody to Raptor.

I. Secondary antibody alone control with DAPI staining.

J. Western blot showing the protein levels of mTOR and Raptor in stimulated and unstimulated embryos (+AA and -AA; 100 embryos per lane). 

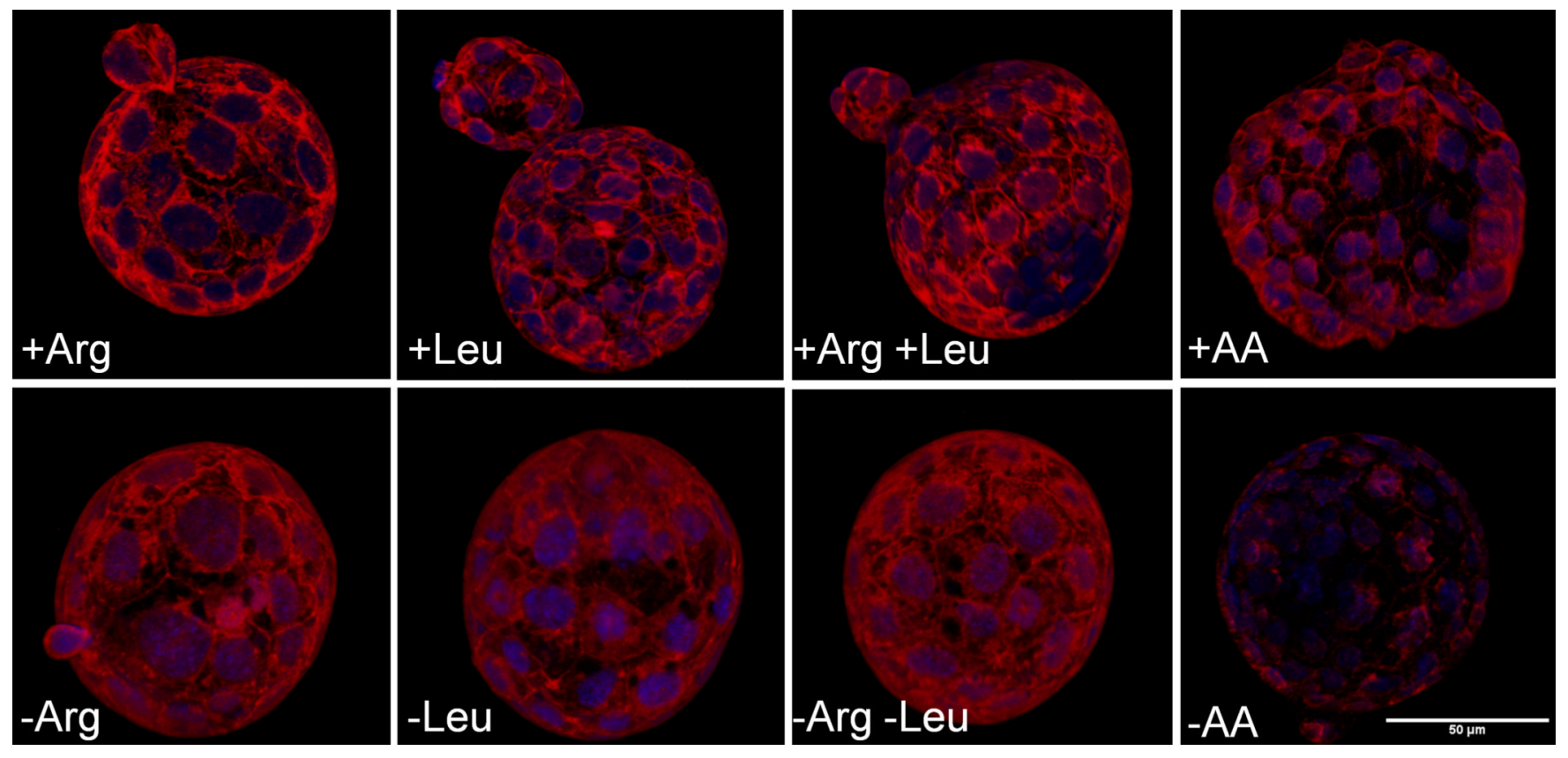

Figure 9. Activated mTOR localization assessed by Cell Signaling Technoly (CST) antibody \#2983 in embryos cultured in various arginine and leucine containing media Increased mTOR signal intensity indicative of an activated mTORC1 in blastocysts after amino acid stimulation in the indicated media. Control is unstimulated blastocyst (-AA, 8 hr) stained for mTOR and DAPI. 

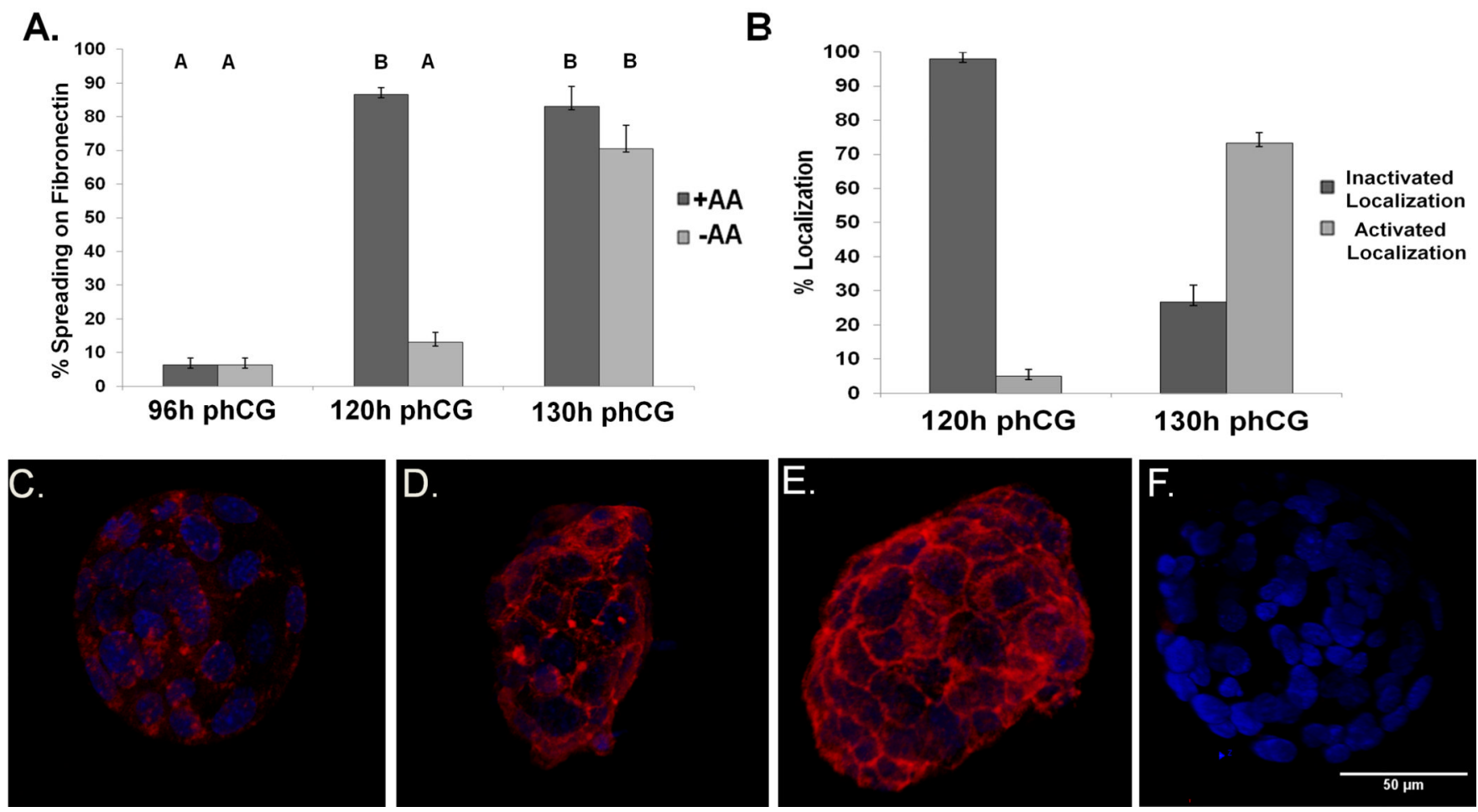

Figure 10. In vivo embryos receive an activation signal between $120 \mathrm{~h}$ and $130 \mathrm{~h}$ phCG

A. Graph showing the percent of embryos flushed from the uterus at 96h, 120h, or $130 \mathrm{~h}$ phCG that form trophoblast outgrowths following culture in either $+\mathrm{AA}$ or -AA medium in vitro. ( $\mathrm{N}=32$ embryos $96 \mathrm{~h}$ phCG $+\mathrm{AA} ; \mathrm{N}=29$ embryos $96 \mathrm{~h}$ phCG -AA; N= 37 embryos $120 \mathrm{~h}$ phCG +AA; N=30 embryos $120 \mathrm{~h}$ phCG $-\mathrm{AA} ; \mathrm{N}=35$ embryos 130 h phCG +AA; $\mathrm{N}=30$ embryos $130 \mathrm{~h}$ phCG $-\mathrm{AA}$ ).

B. Graph showing the percent of blastocysts flushed from the uterus at either $120 \mathrm{~h}$ or 130h phCG that exhibit localization of mTOR in a pattern characteristic of either the inactivated or activated state. ( $\mathrm{N}=9$ embryos $120 \mathrm{~h}$ phCG; $\mathrm{N}=15$ embryos $130 \mathrm{~h}$ phCG)

C. mTOR immunofluoresence of blastocyst flushed from the uterus at $120 \mathrm{~h}$ phCG.

D. mTOR immunofluoresence in a blastocyst flushed from the uterus at $130 \mathrm{~h}$ phCG, characteristic of the inactivated mTOR signal intensity

E. mTOR immunofluoresence in a blastocyst flushed from the uterus at $130 \mathrm{~h} \mathrm{phCG}$, characteristic of the activated mTOR signal intensity

F. Secondary antibody alone with DAPI staining 


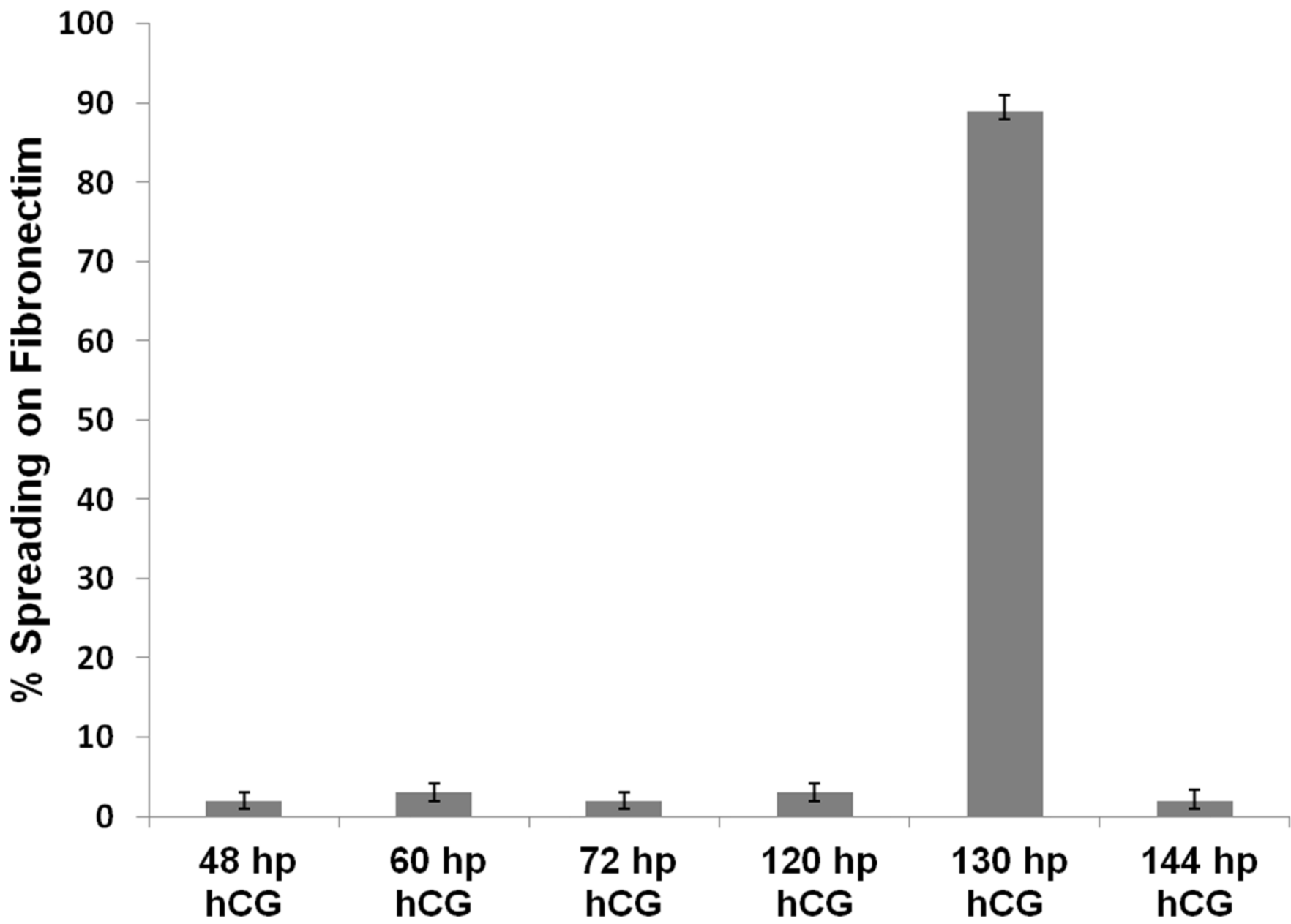

Figure 11. 130h phCG uterine fluid stimulates trophoblast outgrowth Graph showing the percent of embryos exhibiting any trophoblast outgrowth following 24 hours of culture in uterine fluid collected from mice at the indicated stages of pregnancy. Embryos were flushed at $48 \mathrm{~h}$ phCG, cultured in vitro to the blastocyst stage, and used in trophoblast spreading assays when they were at $120 \mathrm{~h}$ phCG. Data represent the mean $+/-$ SEM of 3 independent experiments. $\mathrm{N}=45$ embryos per treatment. 


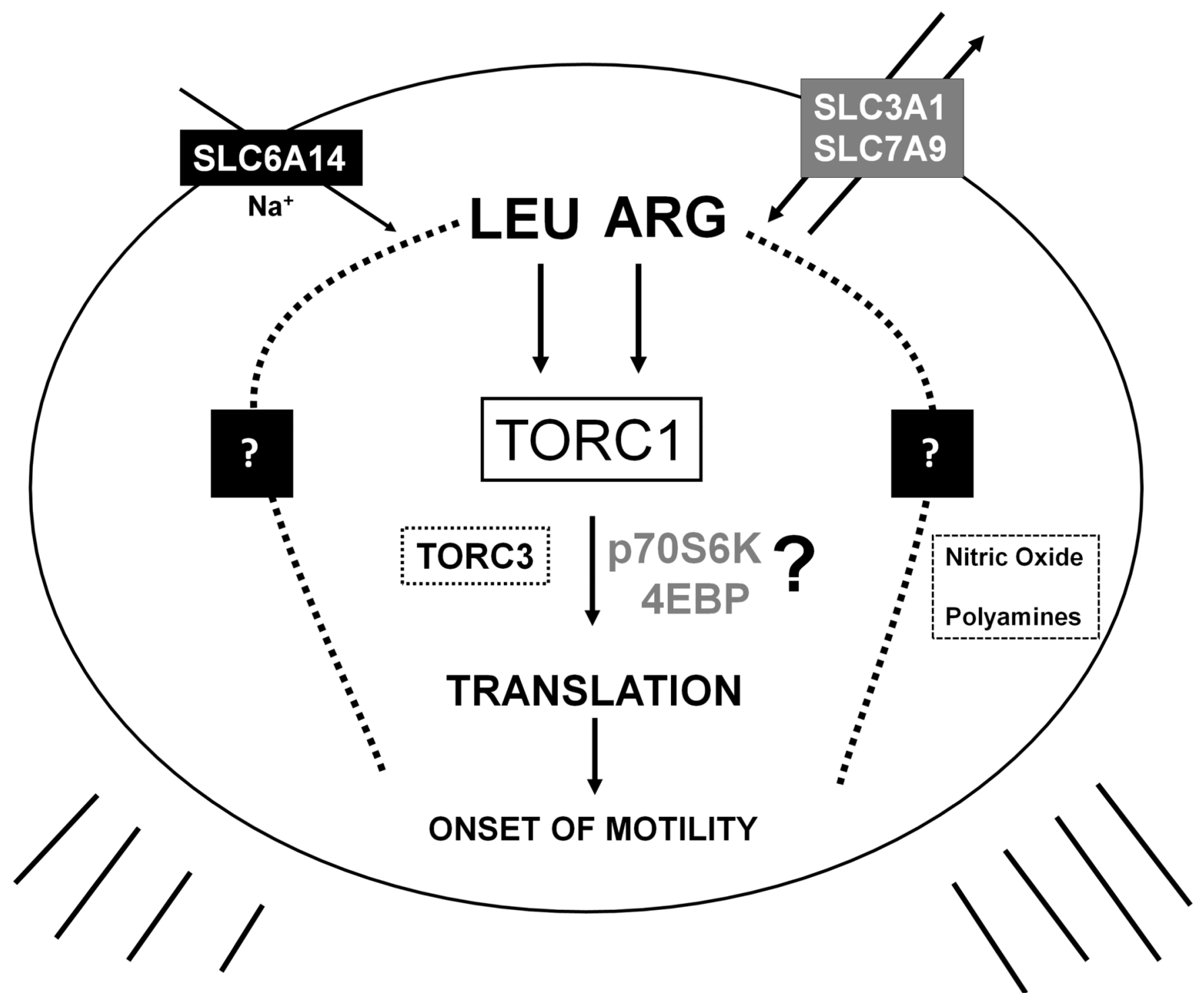

Figure 12.

Model for the action of amino acids on trophoblast cells at the time of implantation. 


\section{Table 1}

Developmental stage of peri-implantation embryos and corresponding hour post hCG (h phCG). Table summarizing the developmental stages of in vitro matured mouse embryos normalized against second superovulatory injection and expressed as hours post hCG (h phCG; hCG: human chorionic gonadotropin)

\begin{tabular}{|l|l|}
\hline $\begin{array}{l}\text { Developmental } \\
\text { Stages }\end{array}$ & $\begin{array}{l}\text { Hours Post } \\
\text { hCG }\end{array}$ \\
\hline 2 Cell & $48 \mathrm{~h} \mathrm{phCG}$ \\
\hline 8 Cell & $60 \mathrm{~h}$ phCG \\
\hline Morulae & $72 \mathrm{~h}$ phCG \\
\hline Cavitating Morulae & $96 \mathrm{~h}$ phCG \\
\hline Early Blastocyst & $120 \mathrm{~h} \mathrm{phCG}$ \\
\hline Mid Blastocyst & $130 \mathrm{~h} \mathrm{phCG}$ \\
\hline Late Blastocyst & $154 \mathrm{~h}$ phCG \\
\hline
\end{tabular}

